\title{
Cc) creative
}

$\begin{array}{llllllllllll}\mathrm{C} & \mathrm{O} & \mathrm{M} & \mathrm{M} & \mathrm{O} & \mathrm{N} & \mathrm{S} & \mathrm{D} & \mathrm{E} & \mathrm{E} & \mathrm{D}\end{array}$

저작자표시-비영리-변경금지 $\mathbf{2 . 0}$ 대한민국

이용자는 아래의 조건을 따르는 경우에 한하여 자유롭게

- 이 저작물을 복제, 배포, 전송, 전시, 공연 및 방송할 수 있습니다.

다음과 같은 조건을 따라야 합니다:

저작자표시. 귀하는 원저작자를 표시하여야 합니다.

비영리. 귀하는 이 저작물을 영리 목적으로 이용할 수 없습니다.

변경금지. 귀하는 이 저작물을 개작, 변형 또는 가공할 수 없습니다.

- 귀하는, 이 저작물의 재이용이나 배포의 경우, 이 저작물에 적용된 이용허락조건 을 명확하게 나타내어야 합니다.

- 저작권자로부터 별도의 허가를 받으면 이러한 조건들은 적용되지 않습니다.

저작권법에 따른 이용자의 권리는 위의 내용에 의하여 영향을 받지 않습니다.

이것은 이용허락규약(Legal Code)을 이해하기 쉽게 요약한 것입니다.

Disclaimer 민 
공학 석사 학위 논문

Various Structural Approaches to Analyze an Aircraft with High Aspect Ratio Wings

고세장비 날개를 가진 항공기의 해석을 위 한 다양한 구조해석 기법에 대한 연구

2013년 2월

서울대학교 대학원

기계항공공학부

멀티스케일 기계설계전공

Anas El Arras 
(3) 서울대학교 
공학 석사 학위 논문

Various Structural Approaches to Analyze an Aircraft with High Aspect Ratio Wings

고세장비 날개를 가진 항공기의 해석을 위

한 다양한 구조해석 기법에 대한 연구

2013년 2월

서울대학교 대학원

기계항공공학부

멀티스케일 기계설계전공

Anas El Arras 


\title{
Abstract \\ Various Structural Approaches to Analyze an Aircraft with High Aspect Ratio Wings
}

\author{
Anas El Arras \\ School of Mechanical and Aerospace Engineering \\ Multiscale Mechanical Design \\ The Graduate School \\ Seoul National University
}

Aeroelastic analysis of an aircraft with a high aspect ratio-wing for medium altitude and long endurance capability was attempted in this thesis. In order to achieve such objective, various structural and aerodynamic models were adopted. The traditional approach has been based on an Euler-Bernoulli beam structural model. In addition to that, a geometrically non-linear beam model was adopted. The structural analysis results of the present beam models were obtained and later compared with those by three-dimensional NASTRAN finite element model. The relevant unsteady aerodynamic forces were acquired by two methods. First, a finite state dynamic inflow unsteady aerodynamics model was developed and evaluated. Second, ZAERO, which is based on the doublet-lattice method, 
was used. These two kinds of aerodynamic forces were compared, and applied to the foregoing flutter analysis. For instance, the structural mode shapes and natural frequencies from both the Euler-Bernoulli beam structural model and the three-dimensional finite element model were transferred to ZAERO, which used the DLM aerodynamics to estimate the flutter. Similarly, flutter prediction was conducted by combining the EulerBernoulli beam structural model and the finite state dynamic inflow unsteady aerodynamics. The next phase of the present research will deal with the analysis of the possible interaction between the rigid-body degrees of freedom and the aeroelastic modes.

Key Words: High aspect-ratio wings, Euler-Bernoulli Beam Structural Model, Three Dimensional Finite Element Model, Geometrically NonLinear Beam Model, Doublet-Lattice Method, Finite State Dynamic Inflow Unsteady Aerodynamics, Flutter Analysis

Student ID: 2011-22890 


\section{Contents}

Chapter 1. Introduction ......................................................................... 1

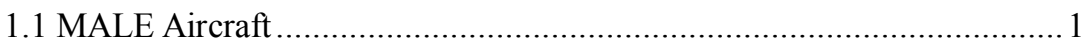

1.2 Background and Motivation...................................................... 4

1.3 Research Objectives and Approach ........................................... 10

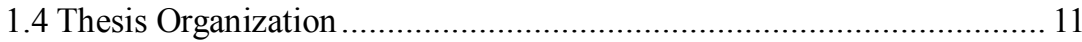

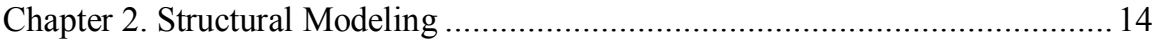

2.1 Euler-Bernoulli Beam Structural Model ............................................... 14

2.2 Three-Dimensional Finite Element Model .......................................... 19

2.3 Geometrically Non-Linear Beam Model.............................................. 21

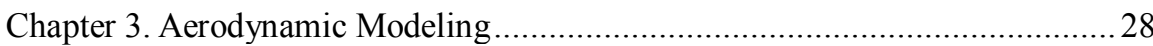

3.1 Doublet Lattice Method (DLM) ................................................. 28

3.2 Finite State Dynamic Inflow Unsteady Aerodynamics ....................... 31

Chapter 4. Flutter Modeling ...................................................................... 38

4.1 Flutter Prediction Based on the Euler-Bernoulli Beam Structural Model

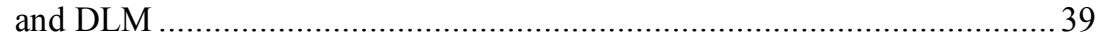

4.1.1 The k-Method .................................................................. 41

4.1.2 The g-Method ............................................................... 42

4.1.3 Wing Flutter Analysis......................................................... 44

4.1.4 Complete Aircraft Flutter Analysis...................................... 45

4.2 Flutter Prediction Based on the Geometrically Non-linear Beam

Model and Finite State Dynamic Inflow Unsteady Aerodynamics.............. 45

4.2.1 Steady-state Response ..................................................... 47

4.2.2 Perturbed Response...................................................... 47

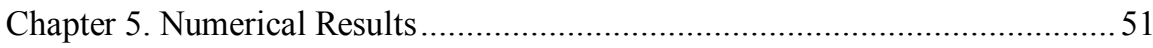

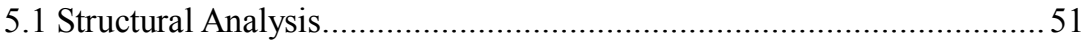

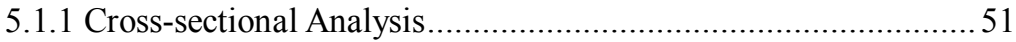

5.1.2 Free Vibration Analysis ........................................................... 52

5.1.3 Mass Density Optimization and Improved

Free Vibration Analysis...................................................... 54

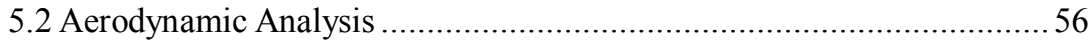

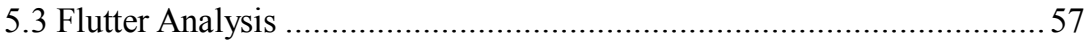

Chapter 6. Conclusion and Future Works................................................. 79

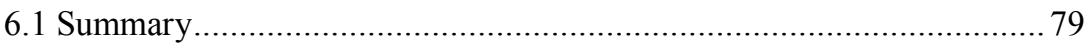

6.2 Future Works .............................................................. 80

- iii - 


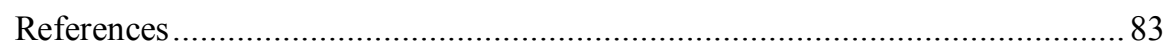

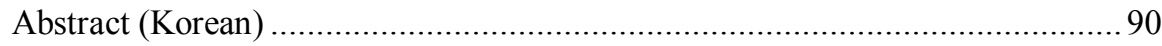

- iv - 


\section{List of Figures}

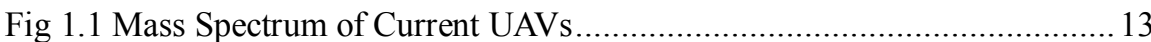

Fig. 2.1 3-D FEM Wing Representation.......................................................... 25

Fig 2.2 Cross-Section at the wing tip and root.......................................... 26

Fig 2.3 Global reference frame a, undeformed beam reference frame $b$ and deformed beam reference frame B.......................................................... 27

Fig 3.1 A Rectangular Lattice ........................................................................ 36

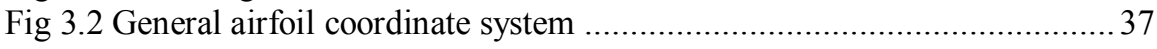

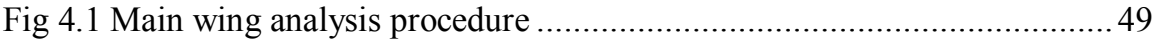

Fig 4.2 Complete aircraft analysis procedure ............................................. 50

Fig 5.1 Cross-section of the present high aspect-ratio wing .............................. 62

Fig 5.2 Mode shapes of the single main wing by the 3-D FEM model predicted by

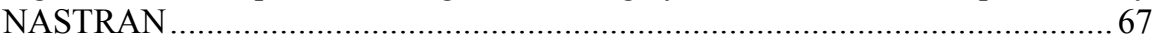

Fig 5.3 Mode shapes of the single tail wing by the 3-D FEM model ...................6 68

Fig 5.4 Mode shapes of the single main wing by the Euler-Bernoulli beam

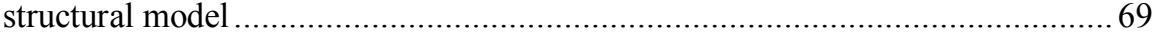

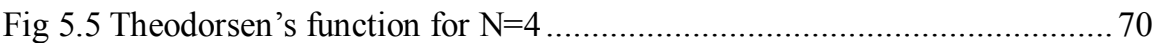

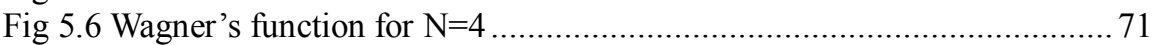

Fig 5.7 Flutter analysis by the EB beam structural model and the FS dynamic

inflow unsteady aerodynamics model for the main wing ............................... 75

Fig 5.8 Damping and Frequency plots by the 3-D FEM and the EB beam structural model for the main wing at M 0.5, non-matched flutter solution........................ 76

Fig 5.9 Damping and Frequency plots by the 3-D FEM model for the tail wing at M 0.5, non-matched flutter solution ..................................................... 77

Fig 5.10 Damping and Frequency plots by the 3-D FEM model for the complete aircraft at M 0.5 , non-matched flutter solution ................................................ 78

\section{List of Tables}

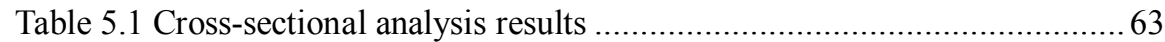

Table 5.2 Natural frequencies of the single main wing ..................................6 64

Table 5.3 Natural frequencies of the single tail wing ....................................65

Table 5.4 Comparison upon the natural frequencies of the single main wing ......66 66

Table 5.5 Main Wing Flutter Prediction Results ............................................... 72

Table 5.6 Tail Wing Flutter Prediction Results .................................................. 73

Table 5.7 Complete Aircraft Flutter Prediction Results...................................... 74 


\section{Chapter 1. Introduction}

\subsection{MALE Aircraft}

An unmanned aerial vehicle, also known as a drone, is an aircraft without a human pilot on board. The flight of the unmanned aerial vehicles (UAVs) can be controlled in several ways, including flight controlled autonomously by computers in the vehicle itself, by a pilot on the ground or in another vehicle, or remotely controlled by a navigator. UAVs are built in a vast array of shapes, sizes, configurations, and defining characteristics suitable to the execution of an increasing number of operations. With the advancements of more elaborate autonomous control mechanisms, UAVs have been capable of fulfilling more complex and demanding tasks since their beginnings as simple, remote-controlled aircraft.

Varying nomenclature is used to differentiate the classes of UAVs and their individual or class-specific purposes, and the terms ascribed to the individual UAVs varies among those employed for military, research, manufacturing, or professional purposes, among others. The various nomenclatures and classes are based on a variety of parameters, including mass, vehicle configuration, designed application, level of autonomy, type 
of operation, or military-level employment. However, there is currently a lack of consensus for classification of civil UAVs, although both the UK and Australia have developed their own classifications between 'small' and 'large' UAVs based on mass [1,2].

To ensure uniformity with current manned aircraft's regulation, UAV classifications were considered with mass as a primary distinguishing characteristic. Performance capabilities were analyzed with respect to mass to determine if natural breakpoints in performance were present, which then allowed classifications to be representative of common operating characteristics.

The five main classifications of current UAVs are 'Micro', 'Mini', 'Tactical', 'Medium Altitude', and 'High Altitude'. 'Medium' and 'High' altitude UAVs are also known as 'medium-altitude long-endurance' (MALE) or 'high-altitude long-endurance' (HALE) UAVs respectively, indicating their ability to stay aloft for long periods of time. Examples of the UAV mass spectrum are shown in Fig 1.1, along with potential classification boundaries.

A medium-altitude, long-endurance unmanned aerial vehicle (MALE UAV) flies at an altitude window of 10,000 to 45,000 feet for extended durations of time, typically 24 to 48 hours. With their advanced WesternStandard, electro-optical payloads, American, Iranian, and Turkish- 
developed MALE UAVs are considered the most advanced in the world. These countries hold a large market share of the MALE UAV market.

Some examples of MALE UAV systems are TAI Anka (Turkey), MQ-1 Predator, Scaled Composites Model 395 (USA), Shahed 129 (Iran), Chengdu Pterodactyl I (China), and DRDO Rustom (India).

Over the past few years there has been a growing need and demand for medium-altitude, long-endurance aircraft. Medium-altitude, longendurance aircraft, abbreviated in the present research as MALE aircraft, is designed for long-range and long-endurance intelligence and surveillance purposes. For instance, MALE aircrafts are suited to execute a broad range of intelligence and surveillance-related tasks, including monitoring environments for nuclear or chemical contamination, target acquisition, reconnaissance missions, aerial refueling, strategic missile defense, cellular telephone relaying, meteorology, weather forecasting, and numerous others. When compared to other aircraft, the use of a MALE aircraft affords great advantages in the execution of these and other related tasks. The many benefits of MALE aircraft are largely the product of their design; for instance, MALE air vehicles are generally smaller than most other manned air vehicles and accordingly cost less to operate, as fuel and hangar costs are much lower. Moreover, the expenditures that are humanrelated are minimal, as the absence of a pilot affords an optimized interior of the UAV because it does not require any seat or complex instrument 
panels requisite for human operation. With the great technical advancements and the ever-increasing amount and complexity of tasks to which they are aptly suited and engaged, the analysis of all aspects of unmanned aerial vehicles has been elevated to an endeavor of utmost importance.

\subsection{Background and Motivation}

One of the most distinguishing features of MALE aircraft design is the incorporation of high aspect-ratio wings, although MALE aircraft are also equipped with medium high aspect-ratio wings. In the present research, high aspect-ratio and flexible wings are considered. These high aspectratio wings, with their long and slender design, are able to maximize the lift-to-drag ratio, but can also be subjected to large deformations under normal operating loads, leading to geometrical nonlinear behaviors. It is because of these possible deformations and nonlinear behaviors that the investigation of the aeroelastic phenomena acting upon high aspect-ratio wings is of utmost importance; understanding the structural behaviors, both linear and nonlinear, of the wings and the unsteady subsonic aerodynamic forces that act upon them is an important facet of understanding the behavior of the aircraft as a whole.

Extensive improvements and developments in the field of high aspect- 
ratio wings in recent decades have merited intensified study and research, quite similarly to the study of fixed rotary-wing aeroelasticity, the drastic improvements of which have also the subject of extensive analysis in recent years. However, whereas the increased study of fixed and rotarywing aeroelasticity heralded the publication of several review articles and studies in the field [3-4], open literature on MALE aircraft remains minimal. The most prominent study related to MALE aircraft with high aspect-ratio wings was conducted by van Schoor et al., which analyzed the aeroelastic properties of high-altitude, long-endurance (HALE) unmanned air vehicles with high aspect-ratio wings [5]. The authors endeavored to study and account for the aeroelastic characteristics and the control of these highly flexible aircraft. Previous investigations in this field employed linearized modes, including rigid body modes, to predict the stability of the aircraft under different flight conditions. Through these studies, it was concluded that unsteady aerodynamics, combined with the flexibility of the aircraft, provided a better depiction of the problem. The importance of the geometrical nonlinearity in HALE aircraft was further analyzed and highlighted in later studies: see [6-8]. The above-mentioned works concluded that the linear analysis based on the undeformed shape may not be accurate in the case of HALE aircraft with high aspect-ratio wings. Furthermore, in reference [9], investigations were carried out to explore the effects of large payload-induced deformations and the effects of 
fuselage parameters and horizontal tails on the flight dynamics of these highly flexible aircraft. Another major undertaking in this work was to account for the resulting increases in sensitivity of some aeroelastic characteristics upon altering configuration parameters. This increased sensitivity was later identified to be the result of the strong coupling of the highly flexible structure and aerodynamics. Given the findings in this work, it becomes apparent that for highly flexible aircrafts such as MALE aircraft, the coupled effects of large deflection due to structural flexibility and flight dynamics (e.g., roll controllability) as well as other aeroelastic effects (e.g., gust response, flutter instability) must and can be properly accounted for by applying a proper aeroelastic formulation.

Determining the dynamics of high aspect-ratio wings has also been the subject of considerable research, both analytical and numerically based. However, these dynamics are difficult to obtain accurately since the deformation in two in-plane and out-of-plane motions and rotation, together with the wing geometry, produce significant nonlinear effects. Hence, many degrees of freedom (DOFs) and higher order nonlinear terms are required in order to create an accurate dynamic modeling of the system. Historically, linear structural models were used for the study of aeroelasticity of aircraft wings [10-11]; however, nonlinearities are present in one form or another. For example, the structural nonlinearity may appear as a spring with free-play, hysteresis, or cubic nonlinearities; these 
types of nonlinearities were investigated by Woolston et al. [12] for a twodimensional airfoil performing pitching and plunging motions. An alternate approach was suggested by Shen [13] using the well-known Kryloff and Bogoliuboff [14] method in nonlinear vibration theory. For moderate deflections, the nonlinear structural equations of a wing in vertical and torsional motion using a second order nonlinear beam theory were given by Hodges and Dowell [15]. This beam theory is characterized by mixed-form formulations, where displacements and strains are both considered as independent variables. Lee and Leblanc [16] described a method for nonlinear aeroelastic analysis without the limitations in the earlier works by using a time-marching finite-difference scheme. In this method, using incompressible aerodynamics, the aeroelastic equations for a two-dimensional airfoil performing plunging and pitching motions were written as a pair of simultaneous finite difference equations. The effect of initial conditions on the nonlinear behavior was studied numerically by varying the displacement from equilibrium of the pitch angle at the start of the airfoil motion. More detailed studies were later carried out by Price et al. [17] who computed power spectral densities, phase space plots, Poincare maps and Lyapunov exponents of the airfoil response to investigate the possibility of chaotic behavior for certain airfoil parameters. Lee et al. [18] investigated the nonlinear aeroelastic response of a twodimensional airfoil considering cubic stiffness nonlinearity in both pitching 
and plunging motions. Mook et al. [19-21] indicated that the aerodynamic nonlinearities alone could be responsible for limit cycle oscillations. Patil et al. [22] used geometrically exact structural analysis and finite state unsteady aerodynamic with stall. In their work, they modified Peter's aerodynamic theory by considering stall corrected expressions to release used theory from any experimental test on a particular airfoil. They showed that stall limits the amplitude of post-flutter unstable oscillations.

Using second order nonlinear beam theory with ONERA dynamic stall model, Tang and Dowell [23-24] compared experimental and theoretical aeroelastic responses of a high aspect-ratio three-dimensional wing with a slender body at the tip and studied the effects of geometric structural nonlinearity and steady angle of attack on nonlinear aeroelastic response of high aspect-ratio wings. Tang and Dowell [25] also demonstrated that the onset of a limit cycle oscillation is dependent upon a delicate balance between stall aerodynamics and structural nonlinear forces.

The importance of aerodynamic and structural geometrical nonlinearities in aeroelastic behavior of high aspect-ratio wings was studied by Patil and Hodges [26] using doublet-lattice aerodynamic theory along with nonlinear finite element beam model. In addition to that, Palacios, et al. [27] have developed an analysis framework based on mixed-form beam theory, which can model slender beams with embedded piezoelectric materials. Displacement-based or mixed-form beam theories 
may be used for different applications with different emphasis. One aspect that should be considered during structural analysis is the compatibility of the selected formulation; for example, if the theory selected for structural modeling and analysis may facilitate the analysis of controls and aerodynamics, this proves to be greatly beneficial in creating a comprehensive analysis. A strain-based beam formulation has also been adopted in previous studies, which was originally developed for the modeling of highly flexible aircraft with embedded active materials. High aspect-ratio wings have been modeled as slender beams, whereas the fuselage was treated as a rigid body. The two-dimensional finite state inflow theory has been used for unsteady aerodynamic modeling [28]. An explicit integration method has been implemented for the time marching solutions. Brown, et al. [29] employed a rigid body free to roll, with a coupled bending and twisting deformation to model the effects of the strain actuation on the behavior of highly flexible high aspect-ratio wings. Cesnik, et al. [30] have introduced flexibility of fuselage and vertical tails to the analysis by following the initial contribution on the strain-based framework. Shearer et al. [31] and $\mathrm{Su}$ [32] have also attempted to analyze the nonlinear flight dynamics and the aeroelastic response and of a HALE aircraft, respectively. The method used by the first endeavored to incorporate low order nonlinear strain-based equations along with unsteady finite state potential-flow aerodynamics. As for the subsequent 
study, the author focused on the investigation of the nonlinear aeroelastic stability with a special emphasis on a trim state-dependent stability boundary.

\subsection{Research Objectives and Approach}

In this research, an aeroelastic analysis of the high aspect-ratio wings of a MALE aircraft will be developed and validated. These aircraft are able to effectively perform medium-altitude, long-endurance surveillance missions largely because of their structural design, such as their long, slender wings, which allow for a maximized lift-to-drag ratio. One of the numerous advantages of the adopted approach is that it encompasses every important aspect of both structural and aerodynamic modeling. In addition, when compared to previous works in the field, it represents a significant advancement in terms of computing times. This research endeavors to develop an accurate analysis of the high aspect-ratio wings through the development of in-house tools that offer significant advancements that ensure the reduction of the problem's complexity while encompassing all its important aspects.

The first order of research is the extraction of the cross-sectional inertia and stiffness properties using the Variational Asymptotic Beam Sectional Analysis (VABS) [33]. With this in mind, the free vibration analysis will 
be carried out and natural frequencies will be obtained, which will allow for an aeroelastic response analysis to be performed with the first few modes. However, MALE aircraft may also undergo large deformations under normal operating loads leading to a geometrically nonlinear behavior. Consequently, nonlinearities will be considered in the last phase of this research. A nonlinear model would facilitate the study of other important aspects of MALE aircraft such as the trim effect. In addition to this, the next phase will also comprise investigations on the effects and inherent consequences of the inevitable interaction between rigid-body flight dynamics and aeroelastic modes.

\subsection{Thesis Organization}

Following this introduction, a range of structural and aerodynamic models used for aeroelastic computations are described separately in Chapters 2 and 3. Chapter 4 integrates the structural and aerodynamic chapters into several flutter analysis models: an Euler-Bernoulli beam structural model with the finite state dynamic inflow unsteady aerodynamics as well as the doublet-lattice method mixed with both the Euler-Bernoulli beam and the three-dimensional finite element structural models. Results from these aeroelastic models focus on the effects of aerodynamics, beam geometry, linearities as well as nonlinearities from 
structural and aerodynamic origins to formulate a prediction of critical aeroelastic flutter behavior. Chapter 5 incorporates all the numerical results and finally Chapter 6 summarizes the contributions of this research and suggests avenues for future work. 


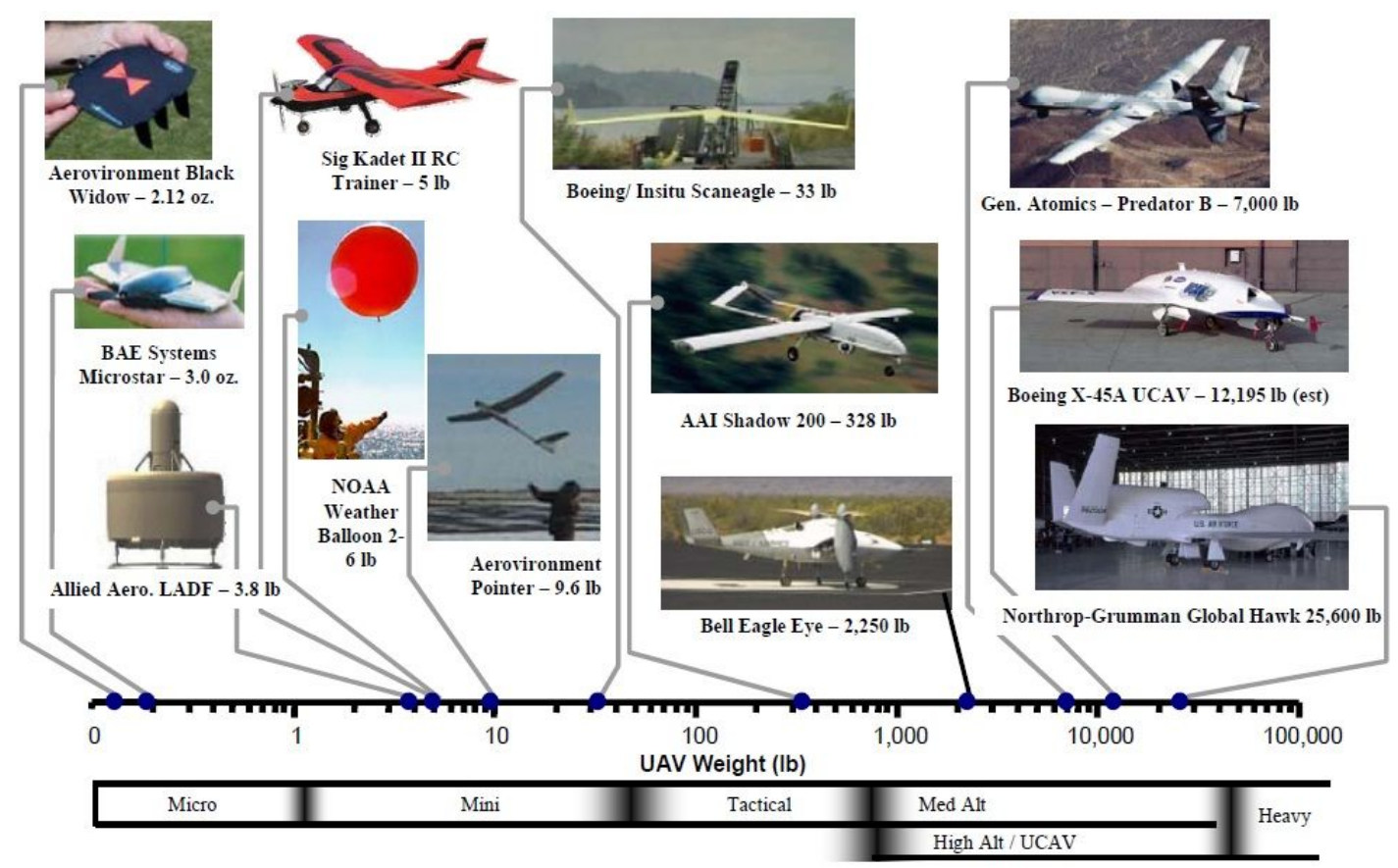

Fig 1.1 Mass Spectrum of Current UAVs

$-13-$ 


\section{Chapter 2. Structural Modeling}

This chapter introduces the three structural models which are employed and analyzed in the present thesis. First, Euler-Bernoulli beam structural model is described. Many studies used a similar formulation, and the present work highlights the features of the structural model developed based on this approach. Secondly, a three-dimensional finite element model, which best describe the complex structure of MALE aircraft, is presented and used to validate the previous model. The third and final model is a geometrically non-linear beam model that has been used previously for rotary wing structural analysis. In this research, the latter model was adopted to cast the non-linear behaviors within the high aspectratio fixed wing.

\subsection{Euler-Bernoulli Beam Structural Model}

At the very beginning of the structural analysis, an Euler-Bernoulli beam structural model, which is displacement-based, was adopted. With this model, linear analysis is very accurate with comparatively small deformations relative to the size of the overall structure and when there are no large torsional effects present. 
The Euler-Bernoulli beam equation arises from a combination of four distinct subsets of beam theory; the kinematic, constitutive, force resultant, and equilibrium definition equations. The outcome of each of these segments is summarized here:

Kinematics: $\quad \kappa=-\theta=-\frac{d w}{d x}$

Constitutive:

$$
\sigma(x, y)=E \varepsilon(x, y)
$$

Resultants:

$$
\begin{gathered}
M(x)=\iint y^{\prime} \sigma(x, y) d y d z \\
V(x)=\iint \sigma_{x y}(x, y) d y d z
\end{gathered}
$$

Equilibrium:

$$
\begin{aligned}
& \frac{d M}{d x}=V \\
& \frac{d V}{d x}=-p
\end{aligned}
$$

While $p$ is the distributed loading acting in the same direction as $y$ (and $w$ ), $E$ is the Young's modulus of the beam. To relate the beam's out-of-plane displacement $w$ to its pressure loading $p$, the results of the four beam subcategories are combined in the order shown below,

$$
\text { Kinematics }=>\text { Constitutive }=>\text { Resultants }=>\text { Equilibrium }=>\text { Beam }
$$

\section{Equation}

First, when one attempts to combine Eqs. (2.5) and (2.6) to eliminate $V$, 


$$
\frac{d^{2} M}{d x^{2}}=-p
$$

Next, by replacing the moment resultant $M$ with its definition in terms of the direct stress $\sigma$,

$$
\frac{d^{2}}{d x^{2}}\left(\iint y \sigma(x, y) d y d z\right)=-p
$$

Using the constitutive relation to eliminate $\sigma$ in favor of the $\operatorname{strain} \varepsilon$, and then using kinematics to replace $\varepsilon$ in favor of the normal displacement $w$,

$$
\begin{aligned}
& \frac{d^{2}}{d x^{2}}\left(E \iint y \varepsilon d y d z\right)=-p \\
& \frac{d^{2}}{d x^{2}}\left(E \frac{d \chi}{d x} \iint y^{2} d y d z\right)=-p \\
& \frac{d^{2}}{d x^{2}}\left(E \frac{d^{2} w}{d x^{2}} \iint y^{2} d y d z\right)=p
\end{aligned}
$$

As a final step, recognizing that the integral over $y^{2}$ is the definition of the beam's area moment of inertia $I, I=\iint y^{2} d y d z$, allows to get the Euler-Bernoulli beam equation,

$$
\frac{d^{2}}{d x^{2}}\left[E I \frac{d^{2} w}{d x^{2}}\right]=p
$$

If $E$ and $I$ do not vary with $x$ along the length of the beam, then the beam equation simplifies to, 


$$
E I \frac{d^{4} w}{d x^{4}}=p
$$

The analysis that has been carried out emphasizes the one-dimensional structural properties of that beam, which was selected for its simplicity and computational speed as opposed to more complex elements such as threedimensional finite element models. The linear behaviors of the high aspect-ratio wings and forces that can act on the flexible wing were also investigated. Generally, these behaviors are examined by modeling the aircraft wing as a structural beam so that this beam theory may be implemented, the analysis of which is afforded while several geometric limitations are placed on the wing. A major limitation of this model has been the inability to accurately determine the limitations on the wing and to clarify the accuracy of the structural analysis method. Bearing this in mind, this research also uses a three-dimensional finite element structural model and will therefore overcome these limitations and serve as a validation tool for the accuracy of the above-mentioned Euler-Bernoulli beam structural model.

As a starting point, the degrees of freedom had to be defined, followed by obtaining mass and stiffness matrices from the minimum energy kinetic principle. Afterwards, a modal analysis study that covers calculation of natural frequencies and mode shapes of the structure is performed. 'Natural frequency' can be defined as the frequency at which free vibration 
of the system can take place and 'Mode Shape' is the deflection of the points on the structure for that mode. Deflection values in the mode shape matrix are not absolute deflections; they should be either normalized to unity or normalized with respect to the mass matrix. Free vibration of a structure can be formulated as given in Eq. (2.12).

$$
[M]\{\ddot{q}\}+[C]\{\dot{q}\}+[K]\{q\}=\{0\}
$$

$\mathrm{M}$ is the mass matrix, $\mathrm{K}$ is the stiffness matrix and $\mathrm{C}$ is the damping matrix of the system. For undamped systems the damping matrix $\mathrm{C}$ equals to zero and Eq. (2.12) reduces to

$$
[M]\{\ddot{q}\}+[K]\{q\}=\{0\}
$$

Assuming harmonic free vibrations, the displacements can be defined as

$$
\{q\}=\{u\} e^{i \omega t}
$$

Substituting in Eq. (2.12)

$$
-\omega^{2}[M]\{u\}+[K]\{u\}=\{0\}
$$

This equation is an eigenvalue problem, the eigenvalues are the squares of natural frequencies and the eigenvectors represent the mode shapes. The mode shapes and modal frequencies of the structure are calculated using the above Euler-Bernoulli beam structural model and are later validated with those from the finite element model. The natural frequencies will be given in Chapter 5. 


\subsection{Three-Dimensional Finite Element Model}

The goal of a structural dynamic analysis includes determining the natural mode shapes and frequencies of an elastic structure in free unforced vibration. The finite element' modeling is well suited for this type of analysis because mode shapes can be accurately derived for geometrically complex structures. The finite elements structural model is typically formulated as an eigensystem, where the eigenvalues and eigenvectors represent the natural frequencies and the mode shapes respectively. The lowest eigenvalues correspond to the lowest characteristic frequencies of the physical system and are typically more useful than the higher modes simply because the physical system tends to experience the lower modes as dominant vibration frequencies.

Therefore, the equations of motion are derived by systematically accounting for the work done by all internal and external generalized forces acting against the corresponding displacements of the system degrees of freedom. The generalized forces contributing to the transfer of work include gravitational, inertial, internal elastic, damping, aerodynamic, distributed, and point forces. The work contributed by each of the generalized forces is equal to the magnitude of the force times the corresponding displacement. The work that is due to distributed forces and moments is integrated over each element based on the assumed 
displacement functions for the finite element. Figure 2.1 shows the threedimensional finite element wing construction.

VABS conducts a modeling of the cross-section of the high aspect-ratio wing. VABS implements a rigorous dimensional reduction from the threedimensional description to a one-dimensional model [33]. The crosssection of a high aspect-ratio wing is presented in Figure 2.2. The inertial and stiffness properties will be presented in the numerical results.

In the free vibration analysis of high aspect-ratio wing structures, the wing structure is equated to a long slender beam capable of bending and torsion. For many classical beam cross-sections, the cross-section is doubly symmetric about both horizontal and vertical axes. This double symmetry leads to an uncoupling of bending and torsional motion. However, even in simple classical wing structures, the cross section has only single symmetry or no symmetry. This loss of cross-sectional symmetry leads to a coupling effect of bending and torsional motion due to an offset of the center of gravity and the center of shear. The resulting coupled equations of motion are dynamically coupled but elastically uncoupled.

In other words, the linear finite element analysis is fairly accurate for a beam that may bend and has small deflections. If large torsional loads are applied, a highly twisted wing is used, or the deflections are large, it may be necessary to use non-linear analysis to obtain an accurate result. 
Moreover, if the material used for the wing is highly flexible, which is the case of high aspect-ratio wings, a linear analysis will not be sufficient. A flexible material will also exhibit coupling between the two transverse bending degrees of freedom which must be accounted for in the offdiagonal terms within the stiffness matrix.

\subsection{Geometrically Non-Linear Beam Model}

The aforementioned explanation highlights the importance of using a geometrically non-linear beam model. A non-linear structural model was introduced in order to incorporate the important effects of nonlinearities in the analysis of a high aspect-ratio wing. The adopted non-linear approach is based on the variational formulation for moving beams in global frame, which has also been used for rotorcraft (especially for the hover condition) [34]. This method involves the implementation of the non-linear beam formulation without any simplifying ordering scheme that affords a much more accurate and all-encompassing analysis of the high aspect-ratio wings. Additionally, the transformation of the beam's natural reference frame to a global frame is achieved while enabling the whole analysis to become more simple and precise without destroying its compactness. Among other indisputable advantages that it provides, this method also ensures that there is no degree of freedom reduction required to determine 
the nonlinearities.

The global frame used along this beam formulation is illustrated on Fig.2.3. Displacement, cross-sectional rotation and all the relevant data are formulated accordingly. The rotation matrix $C=C^{a b} C^{B a}$ is expressed as

$$
C=\frac{\left(1-\frac{\theta^{T} \theta}{4}\right) \Delta-\tilde{\theta}+\frac{\theta \theta^{T}}{2}}{1+\frac{\theta^{T} \theta}{4}}
$$

where $\Delta$ is a $3 \times 3$ identity matrix.

The variational formulation can be written as

$$
\begin{aligned}
& \int_{t_{1}}^{t_{2}} \int_{0}^{1}\left(\delta V_{B}^{* T} P_{B}+\delta \Omega_{B}^{* T} H_{B}-\delta \gamma^{* T} F_{B}-\delta \kappa^{* T} M_{B}+\delta F_{B}^{T}\left(\gamma-\gamma^{*}\right) d x_{1} d t+\right. \\
& \left.\delta M_{B}^{T}\left(\kappa-\kappa^{*}\right)-\delta P_{B}^{T}\left(V_{B}-V_{B}^{*}\right)-\delta H_{B}^{T}\left(\Omega_{B}-\Omega_{B}^{*}\right)\right)+\int_{t_{1}}^{t_{2}} \int_{0}^{1} \overline{\delta W} d x_{1} d t=\overline{\delta W}
\end{aligned}
$$

where $t_{1}$ and $t_{2}$ are arbitrary fixed times, $\overline{\delta A}$ represents the virtual action at the ends of the beam and time interval, and $\overline{\delta W}$ is the virtual work of the applied loads. The asterisked $*$ terms means that they satisfy the geometrically exact equations. Therefore, each term of Eq. (2.17) is transformed to the global frame that was introduced in the previous paragraph.

The external force and moment vectors are denoted by $f_{a}$ and $m_{a}$ respectively. The 'hatted' terms are the boundary values of the 
corresponding quantities. The strain, force, velocity and momentum are related through the following equations

$$
\left\{\begin{array}{l}
F_{B} \\
M_{B}
\end{array}\right\}=[S]\left\{\begin{array}{l}
\gamma \\
\kappa
\end{array}\right\},\left\{\begin{array}{l}
P_{B} \\
H_{B}
\end{array}\right\}=\left[\begin{array}{cc}
m \Delta & 0 \\
0 & I
\end{array}\right]\left\{\begin{array}{l}
V_{B} \\
\Omega_{B}
\end{array}\right\}
$$

where $[\mathrm{S}]$ is a $6 \times 6$ cross-sectional stiffness matrix, $m$ is the mass density per unit length, $I$ is the matrix of cross-sectional mass moments/products of inertia. The stiffness matrix is generally obtained through the crosssectional analysis. Transverse shear stiffness $S_{33}$ and $S_{33}$ are chosen as large numbers since the effect of transverse shear in the one-dimensional model is negligible when applied to slender beams. $S_{44}$ in the [S] matrix includes the trapeze effect, leading to a nonlinear stiffness formulation

$$
S_{44}=S_{44 \text { material }}+\frac{S_{55}+S_{66}}{S_{11}} \bar{F}_{1}
$$

$S_{44 \text { material }}$ represents the torsional stiffness of the unloaded blade and $\bar{F}_{1}$ is the steady-state value of the axial force.

Eqs. (2.16), (2.17), (2.18) and (2.19) represent a description of the fully nonlinear beam model, on which the variation of the energy is expressed in terms of components of displacement and rotation in a global frame.

The theory presented is only applicable to a rotating blade, either cantilevered or simply supported in its present understanding. Consequently, this approach, one that is based on this structural model, 
will be modified in order to facilitate an accurate analysis of a fixed wing with an incoming free stream, which will represent a different air-flow pattern when compared to rotary wings. In summary, the use of the geometrically non-linear beam would provide a much more accurate and precise result. 


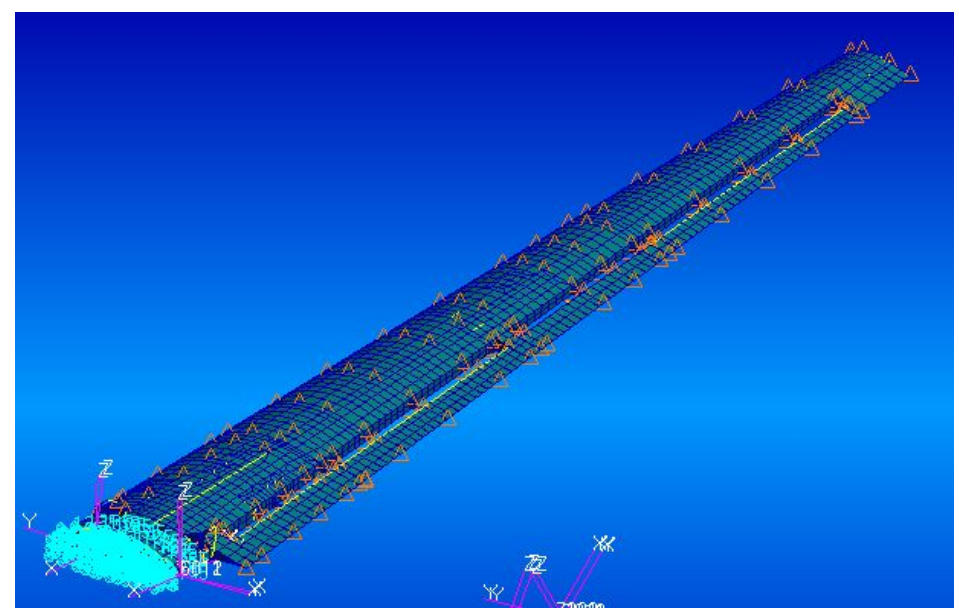

Fig. 2.1 3-D FEM Wing Representation

$-25-$ 


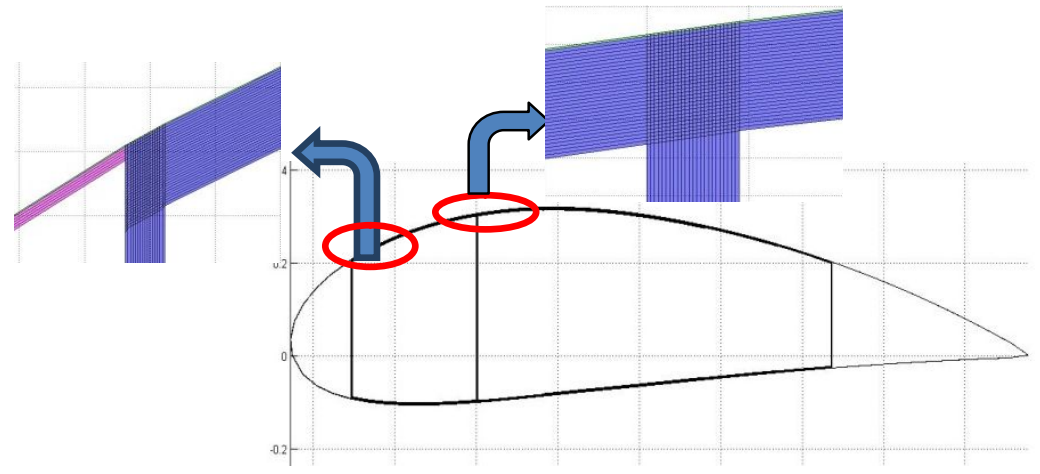

Fig 2.2 Cross-Section at the wing tip and root

$-26-$ 


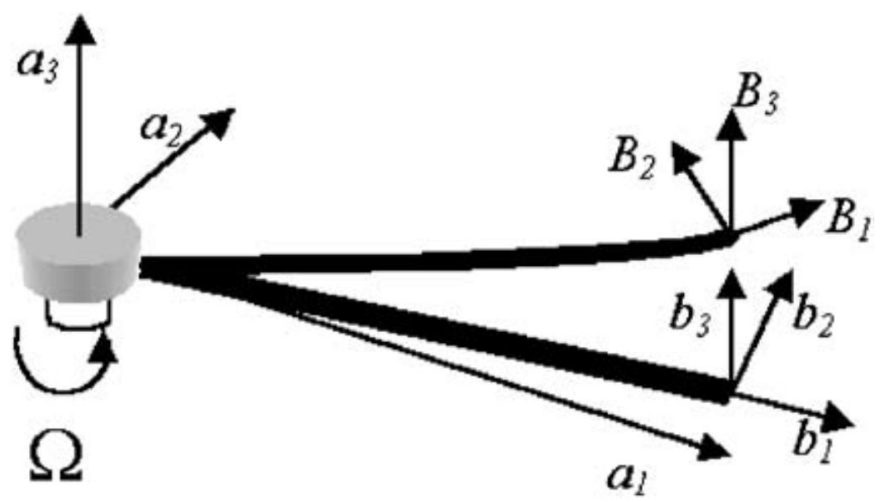

Fig 2.3 Global reference frame a, undeformed beam reference 


\section{Chapter 3. Aerodynamic Modeling}

High aspect-ratio wings reach high aerodynamic efficiency by virtue of their large spans. Except for the regions influenced by the vortices generated at the ends of these wings, the flow over most of the inboard wing section remains two-dimensional. This observation supports the assumption of strip-theory aerodynamics, where the aerodynamic loads at a particular spanwise location are dependent strictly on the geometric angle of attack at that location and are independent of the (threedimensional) aerodynamic influence of other spanwise locations [34].

This chapter identifies a range of aerodynamic models to describe the aerodynamic loads of the wing. The doublet lattice theory is first described to model the aerodynamics of the wing. Subsequent sections discuss the classical finite state dynamic inflow unsteady aerodynamic theory. The latter is validated and computed in an in-house program.

\subsection{Doublet Lattice Method (DLM)}

During the mid to late 1960s, a series of events led to the development of a remarkably unsteady aerodynamic tool called DLM. DLM can be used for interfering lifting surfaces in subsonic flow. A complete description of 
the development of this theory is provided by References [35-37] which are highly recommended to the interested reader. The following general remarks summarize the essential features of the method.

The theoretical basis of the DLM is linearized aerodynamic potential theory. It is also considered as an extension of the steady Vortex-Lattice method to unsteady flow. The undisturbed flow is uniform and is either steady or varying (gusting) harmonically. All lifting surfaces are assumed to lie nearly parallel to the flow. The method consists of dividing the superficies into a certain number of panels (finite elements aerodynamics). The panels are aligned into the direction of the flow and can either be stationary or harmonically non-stationary. Each of the interfering surfaces (or panels) is divided into small trapezoidal lifting elements ("boxes") such that the boxes are arranged in strips parallel to the free stream with surface edges, fold lines, and hinge lines lying on box boundaries. The unknown lifting pressures are assumed to be concentrated uniformly across the onequarter chord line of each box. There is one control point per box, centered span wise on the three-quarter chord line of the box, and the surface normal wash boundary condition is satisfied at each of these points. Fig. 3.1 illustrates the pattern of nine doublet lines for a rectangular wing. The simplicity is that all boxes are treated identically, regardless of its proximity to the wing boundary. 
Let's consider $n$ to be the number of boxes and $\bar{f}_{j} d \mu / 4 \pi \rho$ the doublet strength in the line segment. $\bar{f}_{j}$ is the complex amplitude of the force per unit length along the line, and $d \mu$ represents the incremental length. The normal velocity induced at a point $\left(x_{i}, s_{i}\right)$ on the surface is

$$
w_{i}=\bar{w}\left(x_{i}, s_{i}\right) / U=\sum_{j=1}^{n} \frac{\bar{f}_{j}}{4 \pi \rho U^{2}} \int_{i j} K\left(x_{i}, s_{i}, x_{j}(\mu), s_{j}(\mu)\right) d \mu
$$

where each integration is computed over the line segment of length $l_{j}$. If Eq. (3.1) is applied at $\mathrm{n}$ downwash points on the surface, the $\bar{f}_{j}$ are determined. The force on the doublet line is considered as the force on the box and the pressure difference across the surface is then approximated as follows:

$$
\begin{gathered}
\bar{p}_{j}=\text { force } /(\text { box area })=\bar{f}_{j} l_{j} / \text { (box area) } \\
=\bar{f}_{j} \bar{x}_{j} \cos \lambda_{j}
\end{gathered}
$$

where $\bar{x}_{j}$ is the box average chord and $\lambda_{j}$ represents the sweep angle of the doublet line.

It can be noted that the induced downwash calculated by Eq. (3.2) will be infinite in case the downwash point is located on a doublet line segment or downstream from its end points. Furthermore, the Kutta condition will be satisfied in case each downwash point corresponds to the point at midspan 
and on the three-quarter chord line of a box.

In summary, the three main features of the DLM are as follows; it offers good accuracy for subsonic regimes, it is cost competitive with respect to simpler methods such as strip theories and it enables the analysis of complex geometries as well.

\subsection{Finite State Dynamic Inflow Unsteady Aerodynamics}

Different methods have been used by researchers to efficiently calculate and represent unsteady aerodynamic forces. The early efforts were mainly based on the application of Theodorsen's theory of unsteady twodimensional aerodynamics [38]. However, the direct application of Theodorsen's theory is only limited to the frequency domain analysis and undamped periodic motion, which makes this approach less applicable to control system design and time domain simulation.

Finite-state unsteady models are unsteady aerodynamic approaches which provide more practicality in calculating the unsteady forces and moments [39-40]. Finite state modeling allows one to cast the aerodynamics in the same state-space context as the structural dynamics and controls. This allows the application of advanced time-domain control design techniques to aeroelastic analysis. In Reference 41, the authors developed a two-dimensional finite-state theory that has been the basis for 
calculating the unsteady aerodynamic force by many researchers. The theory calculates aerodynamic loads on a thin-airfoil section undergoing large motions in an incompressible inviscid subsonic flow as a function of kinematic parameters and a set of inflow states. Theoretically, an infinite number of inflow states are required to correctly calculate airfoil loads. However, the desired accuracy can be obtained by using 4 to 8 inflow states. Note that a finite span correction factor has to be applied to account for accurate spanwise force distribution.

In the present research, a finite state unsteady aerodynamics model based on [28] was developed and computed. The use of such a model has several advantages, in terms of computational costs for instance. Besides, there are several other important features of finite state models. First, finite state modeling allows one to cast the aerodynamics in the same state-space context as the structural dynamics and controls. Secondly, the existence of explicit states eliminates the necessity to iterate on solution. This type of finite state aerodynamics offers equations for the induced flow field itself which come directly from the potential flow equations. The states represent induced flow expansion fields rather than velocities. They are hierarchical and consequently, only a few states are needed and the obtained equations are easily coupled with structural equations. For flight simulation of flexible aircrafts, it is necessary to model aerodynamic forces and moments in time domain and express them in motion variables. 
The unsteady aerodynamic forces are derived from the finite-state aerodynamic model that is described in Reference [28]. This finite-state type of aerodynamic analysis is very useful in analyzing aeroelasticity because it can be used in the frequency domain, Laplace domain, or the time domain as desired. This model is used here to form a state-space representation of the aerodynamic problem with a low number of states. Such aerodynamic states are the coefficients of a set of induced-flow expansions.

The general airfoil coordinate system on which this theory is developed is presented in Fig. 3.2

The aerodynamic lift and momentum can be written as:

$$
\begin{gathered}
L=\pi \rho_{\infty} b^{2}\left(-\dot{w}+U_{\infty} \dot{\theta}-b a \ddot{\theta}\right)+2 \pi \rho_{\infty} b^{3}\left(?-\dot{w}+U_{\infty} \theta+b\left(\frac{1}{2}-a\right) \dot{\theta}-\lambda_{0}\right) \\
M=-\pi \rho_{\infty} b^{3}\left(-\frac{1}{2} \dot{w}+U_{\infty} \dot{\theta}-b\left(\frac{1}{8}-\frac{a}{2}\right) \ddot{\theta}\right)
\end{gathered}
$$

where $\lambda_{0}=$ induced-flow velocity and needs to be expressed in terms of airfoil motion. In general, induced-flow theories approximate the effects of shed vortices based on changes they cause in the flow field near the airfoil. Although the induced-flow varies throughout the flow field, one can approximate its value near the airfoil as an average value along the chord line. The induced-flow theory suggested by Peters et al. (1995) does just that, representing the average induced-flow $\lambda_{0}$ in terms of $\mathrm{N}$ induced- 
flow states $\lambda_{1}, \lambda_{2}, \ldots, \lambda_{N}$ as:

$$
\lambda_{0} \approx \frac{1}{2} \sum_{n=1}^{N} b_{n} \lambda_{n}
$$

where $b_{n}$ 's are found by the least-squares method. The induced-flow states $\lambda_{n}$ are obtained by solving a set of $\mathrm{N}$ first-order differential equations approximating the unsteady flow over the airfoil.

$$
[A]\left\{\dot{\lambda}_{n}\right\}+\frac{U_{\infty}}{b}\left\{\lambda_{n}\right\}=\{c\}\left[-\dot{w}+U_{\infty} \theta+b\left(\frac{1}{2}-a\right) \dot{\theta}\right]
$$

where $[\mathrm{A}]$ and $\{\mathrm{c}\}$ are constant matrices and vectors. They are given as follows:

$$
[A]=[D]+\{d\}\{b\}^{T}+\{c\}\{d\}^{T}+\frac{1}{2}\{c\}\{b\}^{T}
$$

where

$$
\begin{aligned}
& D_{n m}=\left\{\begin{array}{c}
\frac{1}{2 n}, n=m+1 \\
-\frac{1}{2 n}, n=m-1 \\
0, n \neq m \pm 1
\end{array} \quad d_{n}=\left\{\begin{array}{r}
\frac{1}{2}, n=1 \\
0, n \neq 1
\end{array}\right.\right. \\
& b_{n}= \begin{cases}(-1)^{n-1 \frac{(N+n-1) ! 1}{(N-n-1) !(n !)^{2}}}, & n \neq N \\
(-1)^{n-1}, & , \quad c_{n}=\frac{2}{n} \\
, & n=N\end{cases}
\end{aligned}
$$


This finite-state model is in a matrix form that allows it to be easily assembled together with the structural model. For finite-span wings, the modified strip theory that extends the two-dimensional aerodynamics to the three-dimensional case is used. As a result, in the above mentioned equations (3.3) and (3.4) for lift and momentum, the following changes should be implemented:

$$
\begin{aligned}
2 \pi & \rightarrow \mathrm{C}_{\mathrm{L} \theta} \equiv \frac{\mathrm{dC}_{\mathrm{L}}}{\mathrm{d} \theta}=\frac{\mathrm{AR}}{\mathrm{AR}+2 \cos \Lambda} 2 \pi \\
\frac{1}{2 \mathrm{~b}} & \rightarrow \mathrm{b}\left(\frac{\mathrm{C}_{\mathrm{L} \theta}}{2 \pi}-\frac{1}{2}\right)
\end{aligned}
$$

The model presented above has been computed and validated. For instance, Theodorson and Wagner functions were used to validate the model, the details of which will be provided in Chapter 5 . 


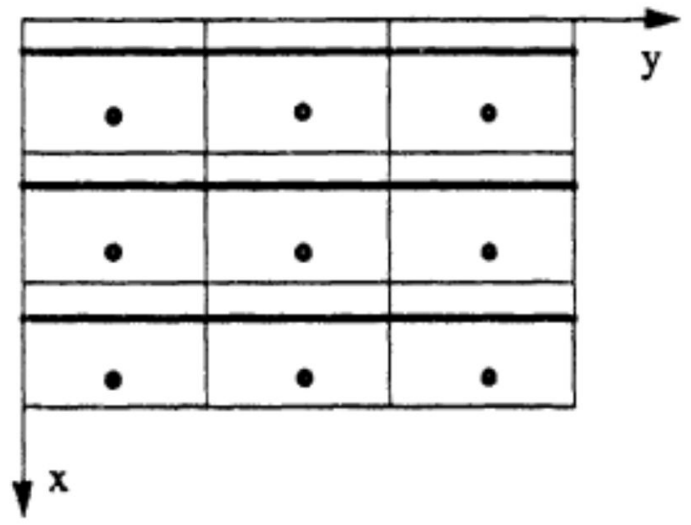

Fig 3.1 A Rectangular Lattice

-36 - 


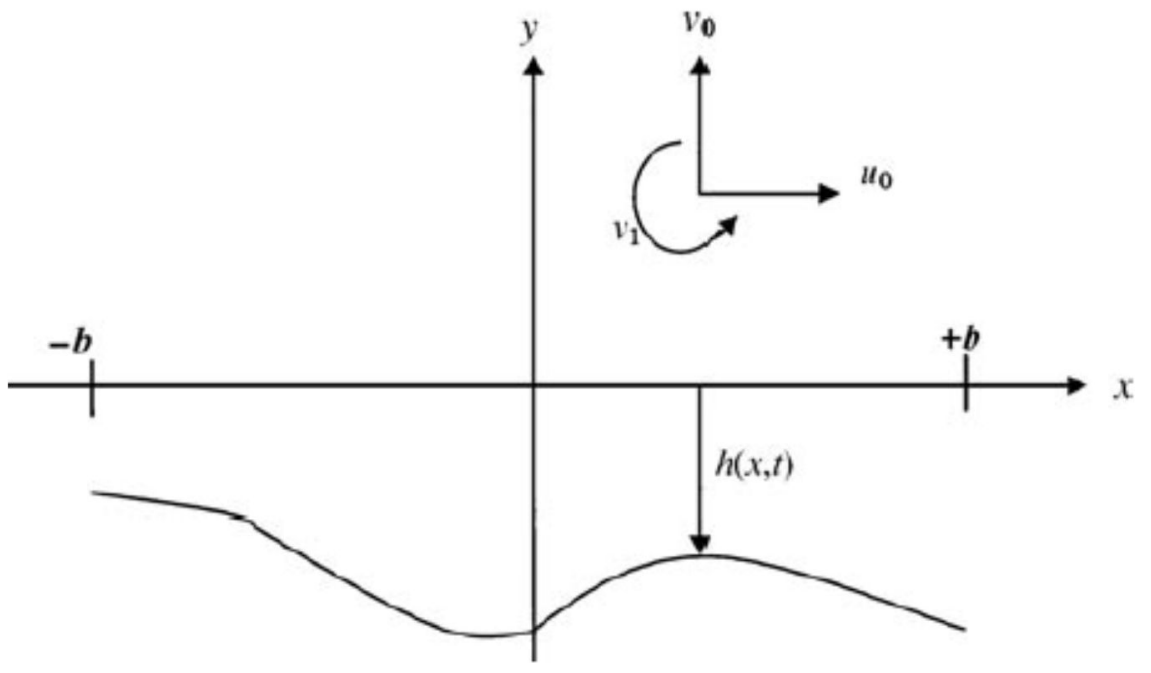

Fig 3.2 General airfoil coordinate system 


\section{Chapter 4. Flutter Modeling}

Flutter is generally observed on wings and control surfaces since these types of structures are subject to large aerodynamic loads. Flutter is produced due to forces that are generated from the dynamic deflections of an elastic structure from the undeformed state [10].

Flutter is defined by a critical speed $U_{F}$ and critical frequency $\omega F . U_{F}$ is the lowest air speed and $\omega F$ is the corresponding circular frequency at which a given structure flying at a given atmospheric condition will undergo sustained, simple, and harmonic oscillations. The solution of flutter leads to a complex eigenvalue problem where two characteristic numbers determine the speed and frequency.

The flutter problem has been modeled in various ways, beginning with single degree of freedom models to CFD - CSD coupled solutions. In this chapter, models proposed by various investigators will be presented in order to explain the development in flutter modeling. The approach based on the Doublet-Lattice Method will be explained. Subsequently, the one based on the combination of the geometrically non-linear beam model and the finite state dynamic inflow unsteady aerodynamics will be elaborated. In the following chapters, the application of these methods along with the 
structural models described in Chapter 2 will be presented.

\subsection{Flutter Prediction Based on the Euler-Bernoulli Beam Structural Model and DLM}

The flight dynamic and aeroelastic system equations of motion can be obtained by combining the structural elastic and unsteady aerodynamic equations. In the present research, for the solution of the aeroelastic system, the steady-state deformation of the wings under given flight conditions is obtained first. The solution provides basic structural data, the distribution of aerodynamic loads in addition to the net forces and moments acting at the body origin. The initial study has been focused on the flutter analysis of the wing using the results by g-Method and k-Method in ZAERO [42], which is based on DLM. In ZAERO, unsteady aerodynamics is estimated upon the structural mode shapes. In other words, two-dimensional panel aerodynamics is applied to the corresponding beam model. Camber and thickness effects with a geometrical shape of an airfoil are then included. The results obtained from the analysis of both the NASTRAN threedimensional finite element model and the Euler-Bernoulli beam structural model, are used in ZAERO to perform the flutter analysis.

The original .F06 files are used to analyze the three-dimensional finite element NASTRAN model. Conversely, the flutter analysis for the Euler- 
Bernoulli beam structural model is based on the .FRE built files. The aerodynamic states are added to the system matrix within ZAERO doubletlattice analysis. Consequently, there will be no clear way to distinguish one from another. In fact, even if it is possible to identify each of the modes, the structural modes were influenced by the aerodynamic lag modes that are automatically implemented throughout the analysis. Therefore, all the modes, including structural and aerodynamic ones, will appear. Given these points, when transferring from NASTRAN to ZAERO, the deformation is linearized in terms of mode shapes and natural frequencies. Thus, while using the Euler-Bernoulli beam structural model, the nonlinearities will likely disappear during the structural analysis.

The flutter matrix equation is derived in the Laplace domain in terms of the generalized mass matrix, $M$, generalized stiffness matrix $K$, and the generalized aerodynamic force matrix $Q$ and given through Eqs. (4.1) and (4.2).

$$
\left[s^{2} M+K-q_{\infty} Q\left(\frac{s L}{V}\right)\right]=0
$$

This can be rewritten as:

$$
\left[\left(\frac{V}{L}\right)^{2} M^{2} p^{2}+K-q_{\infty} Q(p)\right] q=0 ?
$$




\subsubsection{The k-Method}

The basic equation for flutter analysis by the k-Method is:

$$
\left[-\omega^{2} M+\left(1+i g_{s}\right) K-q_{\infty} Q(i k)\right] q=0
$$

It is obtained by replacing $p$ by $i k$ in Eq. (4.2), where $i g_{s}$ is the added artificial complex structural damping that is proportional to the stiffness. Also, the dynamic pressure $q_{\infty}$ can be written as:

$$
q_{\infty}=\frac{1}{2} \rho V^{2}=\frac{1}{2} \rho\left(\frac{\omega L}{k}\right)^{2}
$$

where $\rho$ is the air density.

The k-Method equation can be obtained by substituting Eq. (4.4) into Eq. (4.3) and dividing the resultant equation by $\omega^{2}$

$$
\left[M+\frac{\rho}{2}\left(\frac{L}{k}\right)^{2} Q(i k)-\lambda K\right] q=0
$$

where $\lambda=\frac{\left(1+i g_{s}\right)}{\omega^{2}}$ is the complex eigenvalue of Eq. (4.5).

To solve for these eigenvalue, unsteady aerodynamic computations must be conducted at several reduced frequencies. These reduced frequencies are defined here as the "reduced frequency list". $Q(i k)$ are generated at given Mach number of interest for each reduced frequency. For a given air 
density $\rho$, the eigenvalue of Eq. (4.5) in terms of $\lambda$ 's are solved in the complete reduced frequency list. For $n$ structural modes, there are $n$ eigenvalue corresponding to $n$ modes at each reduced frequency.

The flutter frequency $\omega_{f}$, the airspeed $V_{f}$, and artificial damping $g_{s}$ are given in Eq. (4.6):

$$
\begin{aligned}
& \omega_{f}=\frac{1}{\sqrt{\operatorname{Re}(\lambda)}} \\
& g_{s}=\omega_{f}{ }^{2} \operatorname{Im}(\lambda)=\operatorname{Im}(\lambda) / \operatorname{Re}(\lambda) \\
& V_{f}=\frac{\omega_{f} L}{k}
\end{aligned}
$$

\subsubsection{The g-Method}

The g-Method is a damping perturbation method based. It includes a first order damping term in the flutter equation. This first order term is rigorously derived from the Laplace domain aerodynamics. In the gMethod, it is assumed that a function of $Q(p)=Q(g+i k)$ exists for $g \geq 0, g<0$.

This premise is based on the fact that the Laplace transform of the time domain unsteady aerodynamics for divergent $g>0$ and constant amplitude motions $g=0$ is analytic. Due to this analytical continuation, $Q(p)$ can be extended along the imaginary axis for small $\mathrm{g}$ by means of 
a damping perturbation method:

$$
Q(p)=Q(i k)+\left.g \frac{\partial Q(p)}{\partial g}\right|_{g=0}
$$

The term $\left.\frac{\partial Q(p)}{\partial g}\right|_{g=0}$ in Eq. (4.7) is not available in the k-Method. However, if $Q(p)$ is analytic, it must satisfy the Cauchy-Riemann equations such that:

$$
\begin{aligned}
& \frac{\partial(\operatorname{Re} Q(p))}{\partial g}=\frac{\partial(\operatorname{Im} Q(p))}{\partial k} \\
& \frac{\partial(\operatorname{Im} Q(p))}{\partial g}=-\frac{\partial(\operatorname{Re} Q(p))}{\partial k}
\end{aligned}
$$

Combining Eqs. (4.8) and (4.9) leads to the following general condition:

$$
\frac{\partial \mathrm{Q}(\mathrm{p})}{\partial \mathrm{g}}=\frac{\partial \mathrm{Q}(\mathrm{p})}{\partial(\mathrm{ik})}
$$

Equation (4.10) is valid in the complete p-domain except along the negative real axis where discontinuity due to a branch cut in subsonic flow occurs.

Thus, the term $\left.\frac{\partial Q(p)}{\partial g}\right|_{g=0}$ can be replaced by:

$$
\left.\frac{\partial Q(p)}{\partial g}\right|_{g=0}=\left.\frac{\partial Q(p)}{\partial(i k)}\right|_{g=0}=\frac{d Q(i k)}{d(i k)}=Q^{\prime}(i k)
$$


Since $Q(i k)$ can be provided by the k-domain unsteady aerodynamic methods, $Q^{\prime}(i k)$ can be computed from $Q(i k)$ by a central differencing scheme.

Substituting Eq. (4.11) in Eq. (4.7) gives the approximated p-domain solution of $Q(p)$ in terms of $k$ and for small $g$ :

$$
Q(p)=Q(i k)+g Q^{\prime}(i k)
$$

Substituting Eq. (4.12) in Eq. (4.2) yields the g-Method equation:

$$
\left[\left(\frac{V^{2}}{L^{2}}\right) M p^{2}+K-\frac{1}{2} \rho V^{2} Q^{\prime}(i k) g-\frac{1}{2} \rho V^{2} Q(i k)\right]\{q\}=0
$$

\subsubsection{Wing Flutter Analysis}

In order to analyze the main wing of the aircraft, mass and stiffness matrices were first obtained from the cross-sectional analysis that was carried out using VABS [33]. Afterwards, an evaluation of the structural model of the Euler-Bernoulli beam allowed for the relevant mode shapes and natural frequencies of the beam to be obtained. These properties, as well as the newly-obtained system matrices, were further analyzed and adapted in order to be properly implemented in ZAERO (See Figure 4.1). To analyze the aeroelastic stability of the Euler-Bernoulli beam structural 
model, .FRE files were built and used in ZAERO DLM. Similarly, the three-dimensional NASTRAN finite element model supplied the .F06 files that were utilized for the flutter analysis. The latter was considered as a reference to validate the Euler-Bernoulli beam structural model.

\subsubsection{Complete Aircraft Flutter Analysis}

For the complete aircraft configuration, the assemblage combination of multiple beams was considered. As for the main wing, both mass and stiffness properties were obtained throughout the dimensional reduction carried out by VABS; which constitutes a tool that enables an efficient high-fidelity cross-sectional analysis. Next, the free vibration analysis results for both main and tail wings were achieved from the EulerBernoulli beam structural model's analysis. Later, this outcome was combined with the properties of the fuselage (See Figure 4.2).

Upon compiling all the data, ZAERO was used to perform the flutter analysis, the results of which were again compared with those obtained by the three-dimensional finite element model.

\subsection{Flutter Prediction Based on the Geometrically Non-linear Beam Model and Finite State Dynamic Inflow Unsteady Aerodynamics}

The system equations of the combination of the geometrically non-linear 
beam model presented in Chapter 2 along with the finite state dynamic inflow aerodynamics from Chapter 3 can be presented as follows When combining the above two equations, the indexing system of the finite-state of the inflow model must be associated with the mesh of the structural model.

$$
\begin{aligned}
& F_{s}(X, \dot{X})-F_{L}(X, Y, \dot{X})=0 \\
& F_{I}(Y, \dot{Y})-F_{P}(X, Y)=0
\end{aligned}
$$

Eq. (4.14) is a well-defined aeroelastic formulation for that combination. It includes geometrical exactness for the structural modeling and threedimensionality and unsteadyness for the aerodynamics. The result is presented as a set of nonlinear equations and the solutions for Eq. (4.14) can be written as

$$
\left\{\begin{array}{l}
X \\
Y
\end{array}\right\}=\left\{\begin{array}{l}
\bar{X} \\
\bar{Y}
\end{array}\right\}+\left\{\begin{array}{l}
\breve{X}(t) \\
\breve{Y}(t)
\end{array}\right\}
$$

where $\bar{X}, \bar{Y}$ are the steady-state components of the solution. They are time independent while $\breve{X}(t), \breve{Y}(t)$ are the transient solutions, depend on time. Solutions of the steady-state and perturbed state cases are obtained by taking advantage of the formulation's compactness, as described below. 


\subsubsection{Steady-state Response}

In this case, $\breve{X}(t)=0$, and $\breve{Y}(t)=0$

The system equations Eq. (4.14) become

$$
\begin{aligned}
& F_{s}(X, 0)-F_{L}(X, Y, 0)=0 \\
& F_{I}(Y, 0)-F_{P}(X, Y)=0
\end{aligned}
$$

or simply

$$
\begin{aligned}
& F_{s}(X)-F_{L}(X, Y)=0 \\
& F_{I}(Y)-F_{P}(X, Y)=0
\end{aligned}
$$

The solution can be efficiently calculated by the Newton-Raphson method after calculating the corresponding Jacobian matrix that is analytically obtainable.

$$
[J]=\left[\begin{array}{cc}
\frac{\partial F_{s}}{\partial X}-\frac{\partial F_{L}}{\partial X} & -\frac{\partial F_{L}}{\partial Y} \\
-\frac{\partial F_{P}}{\partial X} & \frac{\partial F_{L}}{\partial Y}-\frac{\partial F_{P}}{\partial Y}
\end{array}\right]
$$

\subsubsection{Perturbed Response}

To investigate the stability of small motions of the system about a steadystate configuration, the system needs to undergo perturbations about and the corresponding equations for the perturbed system are 


$$
\left[\begin{array}{cc}
\frac{\partial F_{s}}{\partial X}-\frac{\partial F_{L}}{\partial X} & -\frac{\partial F_{L}}{\partial Y} \\
-\frac{\partial F_{P}}{\partial X} & \frac{\partial F_{L}}{\partial Y}-\frac{\partial F_{P}}{\partial Y}
\end{array}\right]\left\{\begin{array}{l}
\breve{X} \\
\breve{Y}
\end{array}\right\}+\left[\begin{array}{cc}
\frac{\partial F_{s}}{\partial X}-\frac{\partial F_{L}}{\partial X} & 0 \\
0 & \frac{\partial F_{L}}{\partial Y}-\frac{\partial F_{P}}{\partial Y}
\end{array}\right]\left\{\begin{array}{c}
\dot{X} \\
\dot{\bar{Y}}
\end{array}\right\}=\left\{\begin{array}{l}
0 \\
0
\end{array}\right\}
$$

The flutter can be estimated through applying a set of different velocities and by the evaluation of the damping within the presented aeroelastic responses. 


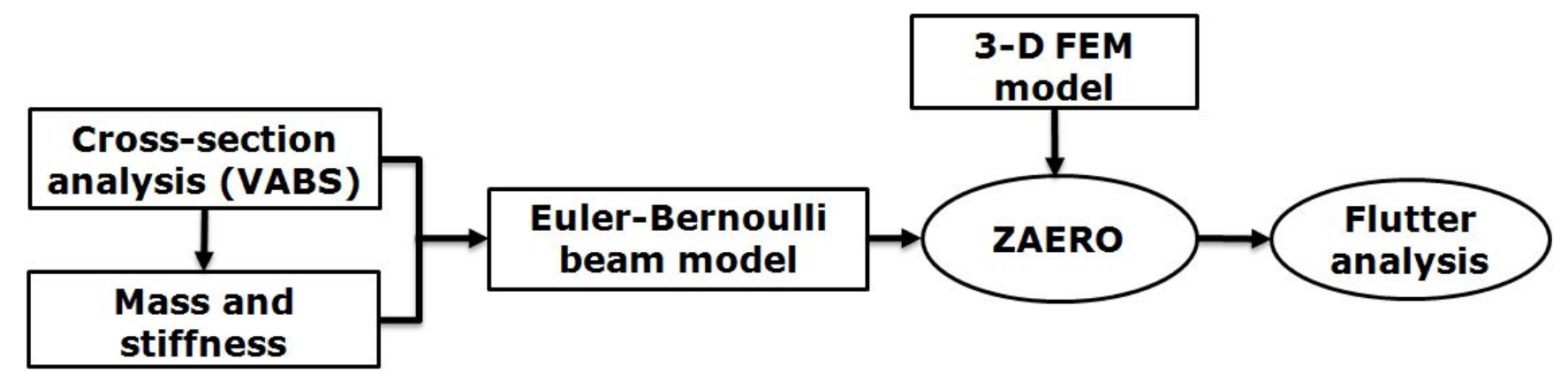

Fig 4.1 Main wing analysis procedure 


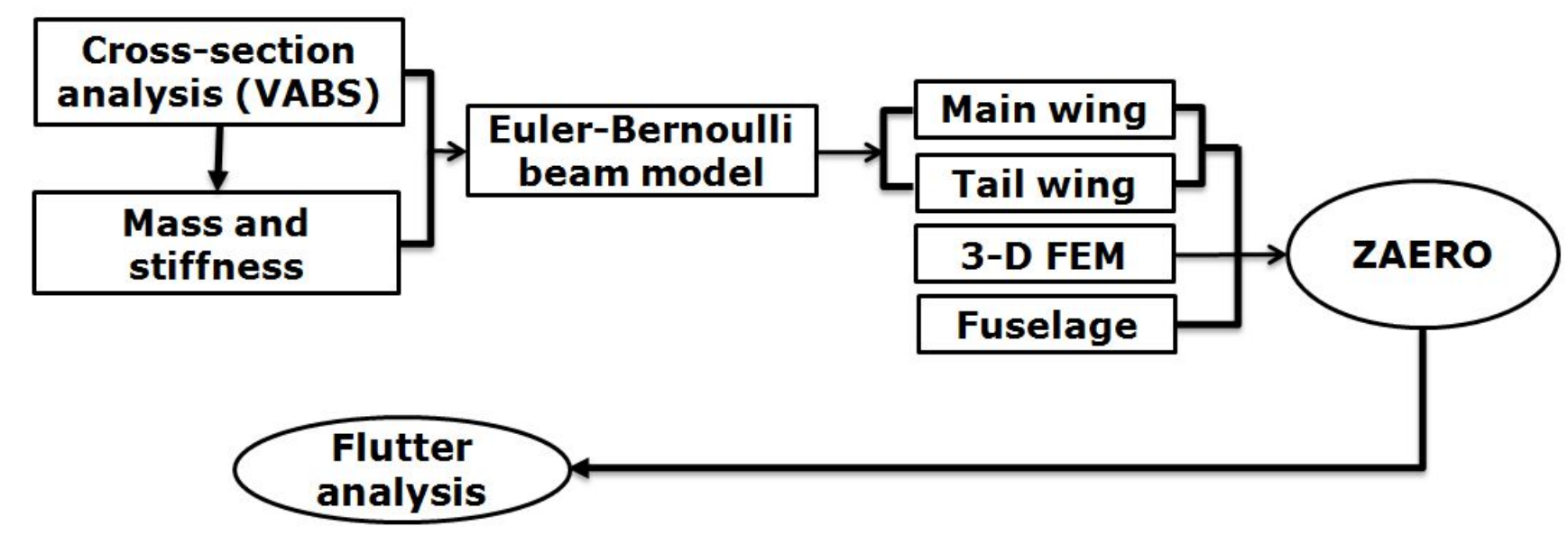

Fig 4.2 Complete aircraft analysis procedure 


\section{Chapter 5. Numerical Results}

The results of this research started with the cross-sectional analysis of the MALE aircraft's using VABS first, and then the relevant mass, inertia and stiffness properties were extracted. Afterwards, the various structural models were analyzed. In other words, both the Euler-Bernoulli beam structural model and the geometrically non-linear beam model were investigated. In addition to that, the three-dimensional finite element model was also evaluated and used a reference. Once those were examined, the according structural models were then coupled with the previously introduced aerodynamics in order to allow for the flutter analysis of the high aspect-ratio wing.

\subsection{Structural Analysis}

\subsubsection{Cross-sectional Analysis}

To begin with, the stiffness and mass results obtained from the threedimensional NASTRAN model are used as inputs within VABS which implements a significant dimensional reduction. The details of the crosssectional geometry and material properties are presented in Figure 5.1.

In Table 5.1, the VABS cross-sectional analysis results of the main wing 
are tabulated. This analysis is capable of providing the appropriate dimensional reduction. Furthermore, it is also able to capture the complex couplings that are present within a composite beam. Generally speaking, the essence of coupling a one-dimensional beam structure to a threedimensional structure is to ensure the compatibility of the displacements at the interface without any loss of accuracy.

\subsubsection{Free Vibration Analysis}

Based on the main three-dimensional finite element model developed in NASTRAN, a more detailed representation of the finite elements for each of the aircraft's components is depicted. Further geometrical and structural properties that are determined through the use of the three-dimensional finite element NASTRAN model are placed into corresponding tables and figures. The structural results from the Euler-Bernoulli beam structural model have been compared to the corresponding three-dimensional finite element results, the latter of which is considered to be a more accurate representation of the actual geometrical and structural data of the aircraft.

In the three-dimensional finite element NASTRAN model, a taper ratio of 0.5 was applied. This is based on the MALE aircraft's wing model that has been used throughout this thesis, which is comprised of 21 ribs and 3 spars. For the structural analysis, about 1,000 grid points are applied.

Using the obtained stiffness and mass properties, the free vibration 
analysis is carried out and natural frequencies are acquired for the high aspect-ratio wing. The relevant natural frequencies are obtained from two models; the Euler-Bernoulli beam structural model and the threedimensional finite element model. In the mean time, the static deflection of the wing is evaluated using the geometrically non-linear beam.

Further analysis is then performed which aims to determine the correlation between the Euler-Bernoulli beam structural model and the three-dimensional finite element model results in terms of mode shapes and natural frequencies.

The natural frequencies obtained from the Euler-Bernoulli beam structural model are presented in Tables 5.2 and 5.3. It can be noted from these tables that the three-dimensional finite element model considers the ribs whereas the natural frequency results within the Euler-Bernoulli beam structural model are obtained by applying mass tuning in order to consider these ribs. Consequently, the first model is considered as being more precise.

In Table 5.2, the structural analysis results of the Euler-Bernoulli beam structural model are presented. In terms of natural frequencies, it shows unsatisfactory correlation with the three-dimensional finite element model illustrated by the significant relative difference in terms of the natural frequencies. 


\subsubsection{Mass Density Optimization and Improved Free Vibration}

Analysis

To clarify, the same mass distribution as the one used throughout the three-dimensional finite element model was also employed for the EulerBernoulli beam structural model's analysis. In other words, the implementation of these mass properties throughout VABS was attempted before performing the Euler-Bernoulli beam structural model free vibration analysis. Consequently, this was suspected to be the cause of the differences noted in the previous results. For that purpose, an iterative procedure has been introduced and used for a more relevant mass distribution. For instance, this iterative procedure was applied based on a new optimized mass density distribution. The results obtained through this new approach show increasingly similar solutions between the EulerBernoulli beam structural model and the three-dimensional model. A comparison between the previous Euler-Bernoulli beam structural model and the improved one is provided through Table 5.4. It can be seen that the relative difference between this improved model and the three-dimensional finite element model is diminishing.

The obtained results indicate that the effect of the number of ribs on the mode shapes, natural frequency and the flutter speed, are quite marginal. Therefore, it can be concluded that changing the number of ribs does not influence the natural frequencies or the flutter speed in any way. Likewise, 
changing the spars would influence the bending and torsion frequencies in addition to the flutter speed that will be further investigated in the next section.

In the meantime, the static deflection of the MALE aircraft's wing was evaluated based on the geometrically non-linear beam model. The crosssectional data was implemented and used to detect any unusual deflection that would occur under certain prescribed loads. The resultant deflection is minimal, which is another factor that confirms the validity of the structural analysis of the wing.

In summary, from a three-dimensional finite element description to an Euler-Bernoulli beam structural model, all the details of the cross-sectional geometry and material properties are included as inputs to calculate both structural and inertial coefficients. Later, these properties can be directly imported into the Euler-Bernoulli beam structural model's analysis to predict the global behavior of the wing. In fact, this imported data is necessary for predicting point-wise three-dimensional distributions of the displacement, strain and stress over the cross-section by VABS (See Figure 5.1). Thereafter, the wing undergoes a mass tuning in each airfoil, leading to the stiffness and mass properties that have been used in the EulerBernoulli beam structural model. Moreover, Table 5.1 indicated that the results obtained through the previous analysis of aforementioned model do not closely match those obtained from the three-dimensional finite element 
model, which is the most accurate. It was concluded that the differences obtained in both mode shapes and natural frequencies might be due to the mass properties used at the very beginning. Therefore, the same mass distribution as the one that has been used to obtain the three-dimensional model was adopted. It was then properly implemented using VABS before analyzing the Euler-Bernoulli beam structural model, which would prove to reduce the disparities among the results of the various structural models.

The vibration mode shapes are obtained through a one dimensional analysis of the Euler-Bernoulli beam structural model. Moreover, ribs undergo a mass tuning since both their mass and stiffness properties could not be modeled in VABS. The mode shapes are presented in Figures 5.2 and 5.3. Later, these mode shapes are compared to the three-dimensional finite element model's data (See Figure 5.4). Through this comparison, the similarities and correspondence between the results in these two structural models becomes increasingly apparent.

\subsection{Aerodynamic Analysis}

One important step that comes next to the structural analysis is the evaluation of the aerodynamics. The latter is very important before going into the flutter analysis. In other words, a proper use of the aerodynamic modeling needs to be validated separately that would confirm the 
exactness of the models on which this study is based. Accordingly, the finite state dynamic inflow unsteady model presented in Chapter 3 has been computed into MATLAB. All the needed equations were defined and implemented, and both Theodorson and Wagner functions were used to validate the accuracy of the model. Those classical functions are plotted in Figures 5.5 and 5.6. The obtained figures clearly illustrate that the in-house built program for finite state dynamic inflow unsteady aerodynamics shows an accurate correlation with the original data enclosed in reference [28].

\subsection{Flutter Analysis}

Upon obtaining the previous results, an aeroelastic analysis is performed using the first six modes of the Euler-Bernoulli beam structural model. One of the key objectives of that analysis is to evaluate the flutter that is expected to occur in a MALE aircraft with high aspect-ratio wings. To facilitate the wing's flutter analysis, several combinations of the structural and aerodynamic models are employed. First, the Euler-Bernoulli beam structural model (EB) along with ZAERO double lattice aerodynamics is used (DLM). The results are obtained by k-Method and g-Method, both of which require the extraction of the generalized mass and stiffness matrices, and the use of the .FRE files. Second, the same Euler-Bernoulli beam 
structural model is combined with the finite state dynamic inflow unsteady aerodynamics model (FS) in MATLAB. The flutter result for the main wing is validated with the three-dimensional finite element model aeroelastic analysis which is based on the NASTRAN .F06 generated files as shown in Table 5.5

The flutter speed for the main wing was determined to be $670 \mathrm{~km} / \mathrm{h}$ when combining the Euler-Bernoulli beam structural model and ZAERO doublet-lattice method. As for the same structural modal along with the finite state dynamic inflow unsteady aerodynamics model, this speed was equal to $640 \mathrm{~km} / \mathrm{h}$ (See Figure 5.7). This was later validated with the results produced by the three-dimensional finite element model's flutter. The difference between the Euler-Bernoulli structural beam model and the three-dimensional finite element model, in terms of the main wing flutter speed result, was found to be less than $10 \%$ in both the cases described above. Besides, the dominant structural mode of the main wing flutter was found to be the first in-plane bending mode in the three-dimensional finite element model. In terms of the flutter frequencies, a difference of $8 \%$ was noticed for the main wing when using the finite state dynamic inflow unsteady aerodynamics model. By analyzing the plots corresponding to the main wing (See Figure 5.8), there was a mode whose imaginary root slightly went across the horizontal axis. It has been identified as being the $8^{\text {th }}$ mode. It coincides with the aerodynamic lag mode added throughout 
ZAERO's aeroelastic analysis and that has a zero frequency. Consequently, it was anticipated that instability would occur at the corresponding flight speed.

In Table 5.6, the results pertaining to the tail wing are presented. The flutter speed for the tail wing was determined to be $792 \mathrm{~km} / \mathrm{h}$ when the Euler-Bernoulli beam structural model and doublet-lattice method were combined in ZAERO. The difference between the Euler-Bernoulli beam structural model and the three-dimensional finite element model, in terms of the tail wing flutter speed result, was found to be approximately $2 \%$. The dominant structural mode of tail wing flutter was assimilated as the first out-of-plane bending mode in the three-dimensional finite element prediction. As for the flutter frequencies, the obtained difference was lower than $30 \%$. From the plots related to the tail wing (See Figure 5.9), an abrupt behavior of one of the modes was noticed. It was identified as the 5th mode, which is, related to the first aerodynamic lag mode. Moreover, it corresponds to zero frequency and thus, it is possible that the tail wing undergoes a single degree of freedom flutter.

As for the entire aircraft, the flutter result from the three-dimensional finite element model is presented in Table 5.7. It was observed that the flutter speed in this case is lower than the one predicted in both Tables 5.5 and 5.6. Also, significant frequency coalescence was found in this case, unlike the previous main-wing only configuration (See Figure 5.10). This 
corresponds to the $14^{\text {th }}$ structural mode. The frequency coalescence that occurred between the $13^{\text {th }}$ and $14^{\text {th }}$ structural modes can be clearly noticed. The damping of the above mentioned modes goes over the horizontal axis, which corresponds to the flutter speed (See Figure 5.10 (c) - (d).). The aforementioned was identified as the interaction of two different modes, one that is related to the tail wing and the other that is from the fuselage. The latter explains that the coalescence that takes place throughout the entire aircraft configuration could be the product of the behaviors of the combined aircraft components' interaction. One important aspect that cannot be determined without this combination is the anti-symmetric coupling that is caused by the pairing of both wings. Furthermore, results indicated that the instability in the main-wing only configuration corresponds to a single degree of freedom flutter, whereas significant frequency coalescence was found in the complete aircraft's case.

In summary, the flutter determined by the present Euler-Bernoulli beam structural model shows similar and correlating flutter speed and frequency results with those determined by the three-dimensional finite element model. Using different structural and aerodynamic models has proved to be a very efficient way to provide a straightforward and detailed analysis of the high aspect-ratio wing. The analysis simplifies the complex problem and provides a wide array of approaches to properly depict the different phenomena related to the MALE aircraft. Furthermore, the newly 
implemented geometrically non-linear beam model will need to be further investigated and properly coupled with the relevant aerodynamics and this would allow for another validation tool of the previous aeroelastic analyses.

$-61-$ 


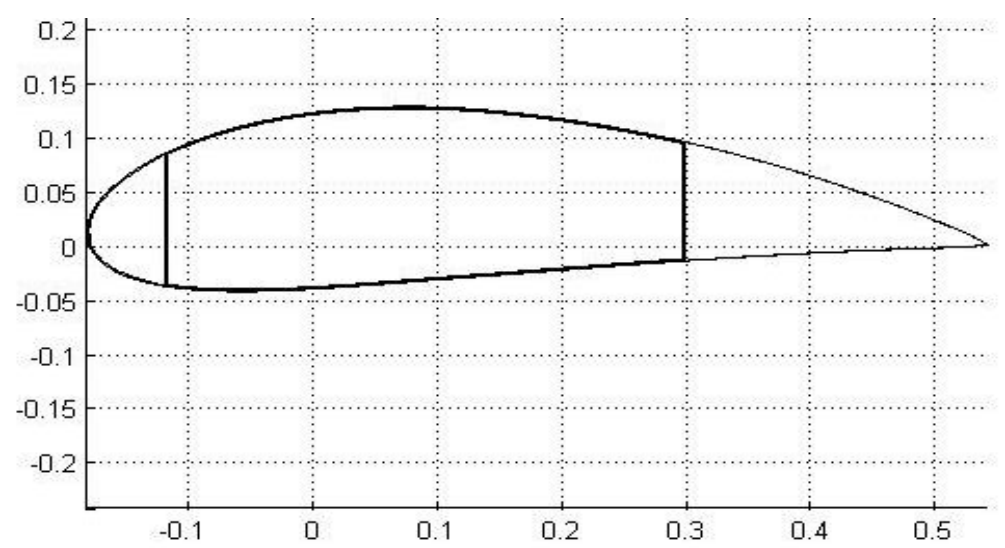

Fig 5.1 Cross-section of the present high aspect-ratio wing

$-62-$ 
Table 5.1 Cross-sectional analysis results

\begin{tabular}{|c|c|c|}
\hline & Main wing root & Main wing tip \\
\hline EA (N-m) & $1.25 \times 10^{9}$ & $7.48 \times 10^{8}$ \\
\hline$E_{\text {out-of-plane }}\left(\mathrm{N}-\mathrm{m}^{2}\right)$ & $2.84 \times 10^{7}$ & $3.25 \times 10^{6}$ \\
\hline EI $I_{\text {in-plane }}\left(\mathrm{N}-\mathrm{m}^{2}\right)$ & $3.44 \times 10^{7}$ & $3.40 \times 10^{6}$ \\
\hline GJ (N-m $\left.{ }^{2}\right)$ & $1.59 \times 10^{8}$ & $1.67 \times 10^{7}$ \\
\hline $\operatorname{Mass}(\mathrm{Kg} / \mathbf{m})$ & 26.48 & 18.864 \\
\hline$I_{x x}(K g-m)$ & 4.51 & 0.582 \\
\hline$I_{y y}(K g-m)$ & 0.70 & 0.078 \\
\hline$I_{z z}(K g-m)$ & 3.80 & 0.504 \\
\hline
\end{tabular}


Table 5.2 Natural frequencies of the single main wing

\begin{tabular}{|l|c|c|c|}
\hline & EB Beam to 3-D FEM Ratio & Relative difference & Mode \\
\hline $\mathbf{1}$ & $5.40: 4$ & $34.8 \%$ & $\mathbf{1}^{\text {st }}$ out-of-plane bending \\
\hline $\mathbf{2}$ & $4.25: 4$ & $6.5 \%$ & $\mathbf{1}^{\text {st }}$ in-plane bending \\
\hline $\mathbf{3}$ & $5.40: 4$ & $35.4 \%$ & $\mathbf{2}^{\text {nd }}$ out-of-plane bending \\
\hline $\mathbf{4}$ & $5.10: 4$ & $28.5 \%$ & $\mathbf{2}^{\text {nd }}$ in-plane bending \\
\hline $\mathbf{5}$ & $4.56: 4$ & $14.2 \%$ & $\mathbf{3}^{\text {rd }}$ out-of-plane bending \\
\hline
\end{tabular}


Table 5.3 Natural frequencies of the single tail wing

\begin{tabular}{|c|c|c|c|}
\hline & EB Beam to 3-D FEM Ratio & Relative difference & Mode \\
\hline $\mathbf{1}$ & $3.48: 4$ & $13.0 \%$ & $\mathbf{1}^{\text {st }}$ out-of-plane bending \\
\hline $\mathbf{2}$ & $3.76: 4$ & $5.84 \%$ & $\mathbf{1}^{\text {st }}$ in-plane bending \\
\hline $\mathbf{3}$ & $4.09: 4$ & $2.4 \%$ & $\mathbf{2}^{\text {nd }}$ out-of-plane bending \\
\hline $\mathbf{4}$ & $4.33: 4$ & $7.6 \%$ & $\mathbf{1}^{\text {st }}$ torsion \\
\hline
\end{tabular}


Table 5.4 Comparison upon the natural frequencies of the single main wing

\begin{tabular}{|c|c|c|c|c|c|}
\hline & $\begin{array}{c}\text { EB beam } \\
\text { to 3-D FEM } \\
\text { Ratio } \\
\text { (With Optimized } \\
\text { Mass Density) } \\
\end{array}$ & $\begin{array}{c}\text { Relative } \\
\text { difference }\end{array}$ & $\begin{array}{c}\text { EB beam } \\
\text { to 3-D FEM } \\
\text { Ratio } \\
\text { (Without Optimized } \\
\text { Mass Density) } \\
\end{array}$ & Relative difference & Mode \\
\hline 1 & $4.45: 4$ & $10 \%$ & $5.40: 4$ & $34.8 \%$ & $\begin{array}{l}1^{\text {st }} \text { out-of-plane } \\
\text { bending }\end{array}$ \\
\hline 2 & $3.51: 4$ & $10 \%$ & $4.25: 4$ & $6.5 \%$ & $\begin{array}{c}1^{\text {st }} \text { in-plane } \\
\text { bending }\end{array}$ \\
\hline 3 & $4.27: 4$ & $6.8 \%$ & $5.40: 4$ & $35.4 \%$ & $\begin{array}{c}2^{\text {nd }} \text { out-of-plane } \\
\text { bending }\end{array}$ \\
\hline 4 & $4.07: 4$ & $1.8 \%$ & $5.10: 4$ & $28.5 \%$ & $\begin{array}{c}2^{\text {nd }} \text { in-plane } \\
\text { bending }\end{array}$ \\
\hline 5 & $3.75: 4$ & $6 \%$ & $4.56: 4$ & $14.2 \%$ & $\begin{array}{c}3^{\text {rd }} \text { out-of-plane } \\
\text { bending }\end{array}$ \\
\hline
\end{tabular}




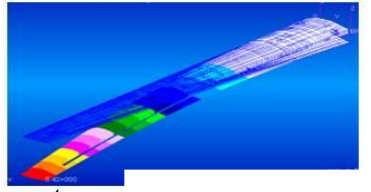

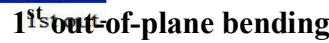

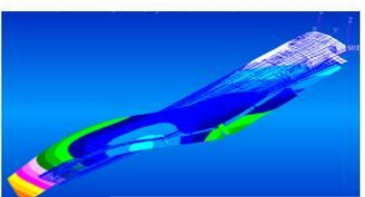

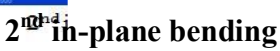

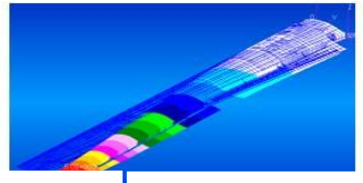

$1^{\text {st }}$ in-plane bending

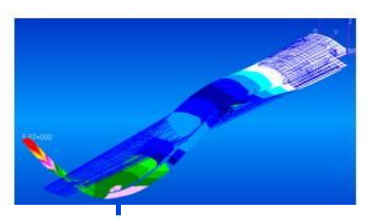

$3^{\text {rd }}$ out ${ }^{\mathrm{t}}$ of-plane bending

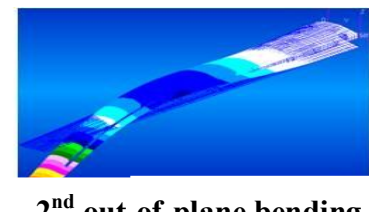

$2^{\text {nd }}$ out-of-plane bending

Fig 5.2 Mode shapes of the single main wing by the 3-D FEM model

$-67-$ 


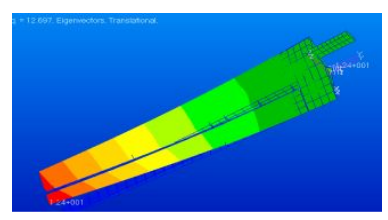

$1^{\text {st }}$ bending

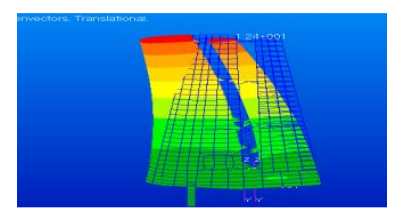

$1^{\text {st }}$ lag

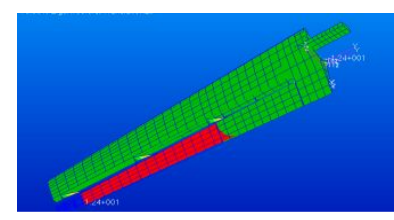

$1^{\text {st }}$ rudder

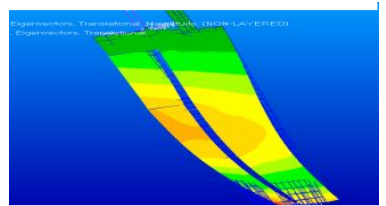

$2^{\text {nd }}$ bending

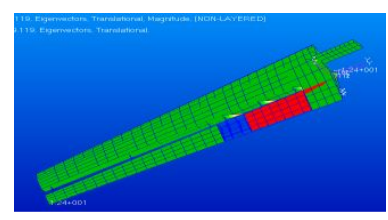

$2^{\text {nd }}$ rudder

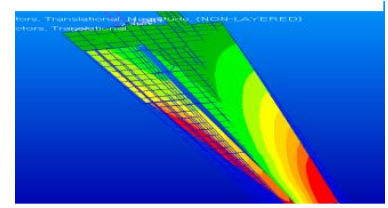

$1^{\text {st }}$ torsion

Fig 5.3 Mode shapes of the single tail wing by the 3-D FEM model

$-68-$ 


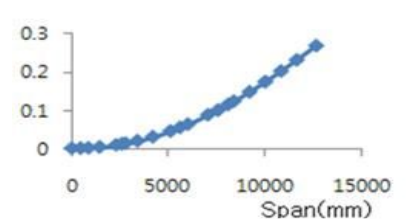

${ }^{1} \mathbf{p}^{\text {st }}$ ottt-of-plane bending

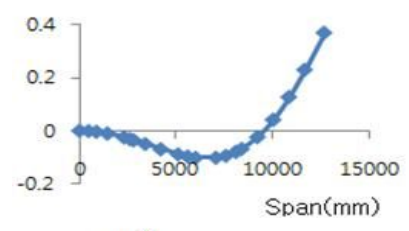

$\mathbf{2}^{\text {nd }}$ iim-plane bending

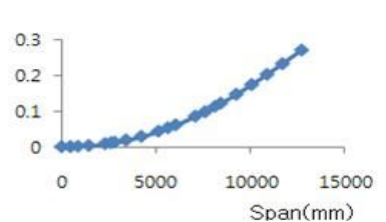

$1^{\text {st }}$ in-plane bending

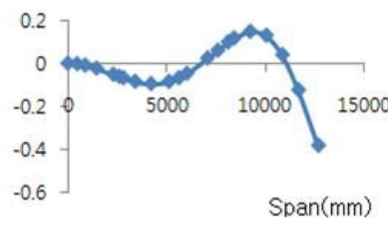

$3^{\text {rd }}$ out-of-plane bending

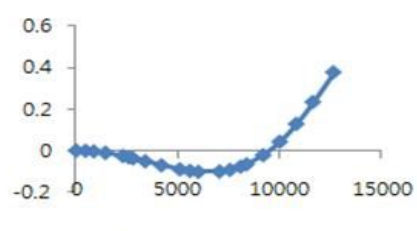

$2^{\text {nd }}$ out-of-plane bending

Fig 5.4 Mode shapes of the single main wing by the Euler-Bernoulli beam structural model 


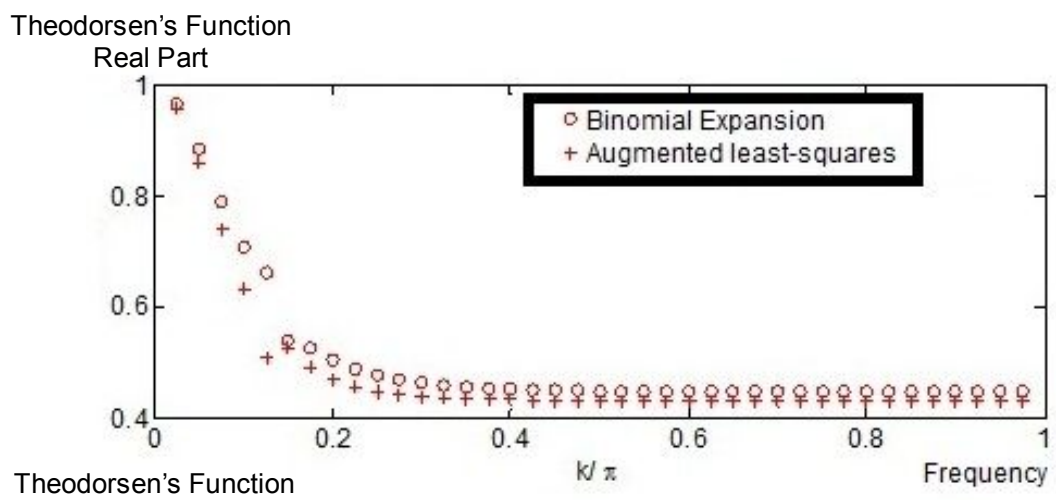
Imaginary Part

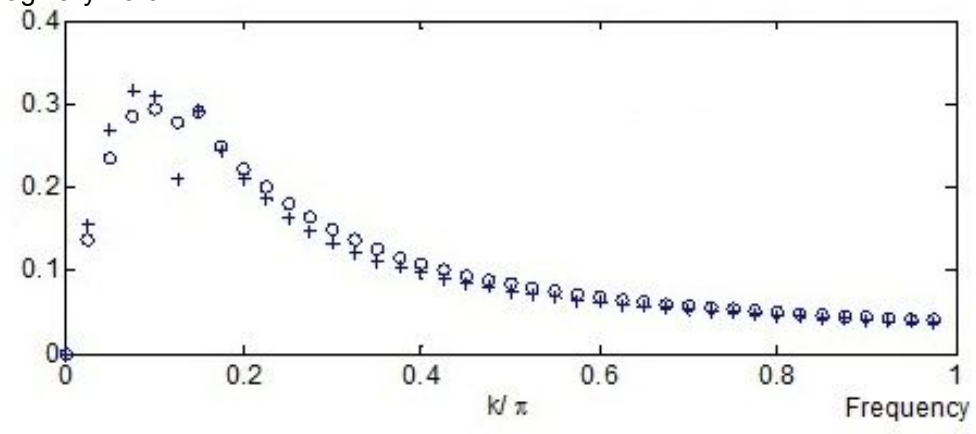

Fig 5.5 Theodorsen's function for $N=4$ 


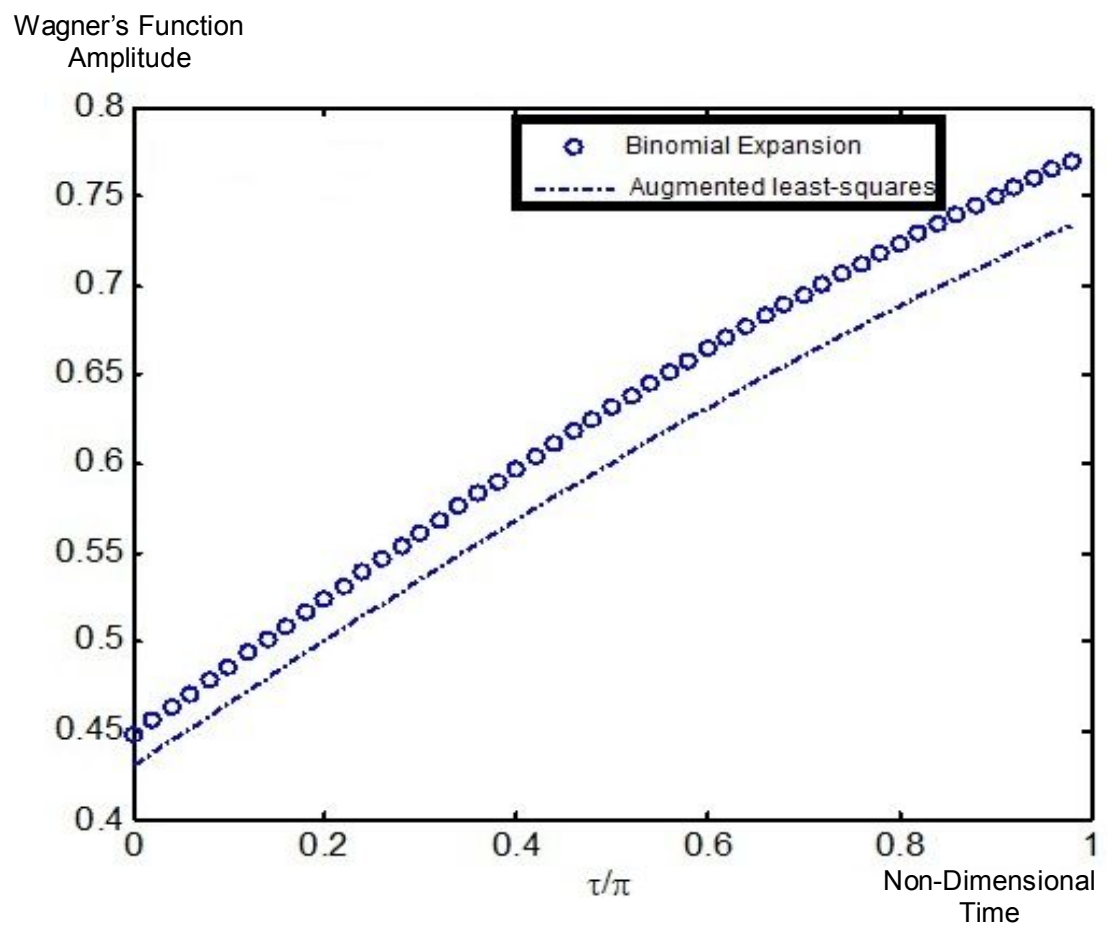

Fig 5.6 Wagner's function for $\mathrm{N}=4$ 
Table 5.5 Main Wing Flutter Prediction Results

\begin{tabular}{|c|c|c|c|c|c|c|}
\hline \multirow[b]{2}{*}{ Flutter } & \multicolumn{2}{|c|}{ 3-D FEM + DLM } & \multicolumn{2}{|c|}{ EB Beam + DLM } & \multirow[t]{2}{*}{$\mathbf{E B}+\mathbf{F S}$} & \multirow[t]{2}{*}{$\begin{array}{c}\text { Minimal Relative } \\
\text { Difference }\end{array}$} \\
\hline & g-Method & k-Method & g-Method & k-Method & & \\
\hline Speed $(\mathbf{k m} / \mathbf{h})$ & 728.4 & 723.9 & 670.3 & 651.6 & 640 & $7.90 \%$ \\
\hline Frequency (Hz) & 6.61 & 6.61 & 10.37 & 10.37 & 7.10 & $7.80 \%$ \\
\hline
\end{tabular}


Table 5.6 Tail Wing Flutter Prediction Results

\begin{tabular}{|c|c|c|c|c|}
\hline \multirow{2}{*}{ Flutter } & \multicolumn{2}{|c|}{$\begin{array}{c}\text { 3-D FEM } \\
+ \\
\text { DLM }\end{array}$} & $\begin{array}{c}\text { EB Beam } \\
+ \\
\text { DLM }\end{array}$ & $\begin{array}{c}\text { Minimal } \\
\text { Relative } \\
\text { Difference }\end{array}$ \\
\cline { 2 - 5 } & g-Method & k-Method & g-Method & $2 \%$ \\
\hline Speed (km/h) & 810 & 795.6 & 792 & $29 \%$ \\
\hline Frequency (Hz) & 19.3 & 19.3 & 25 & 29 \\
\hline
\end{tabular}


Table 5.7 Complete Aircraft Flutter Prediction Results

\begin{tabular}{|c|c|c|}
\hline \multirow{2}{*}{ Flutter } & \multicolumn{2}{|c|}{ 3-D FEM + DLM } \\
\cline { 2 - 3 } & g-method & 648 \\
\hline Speed $(\mathbf{k m} / \mathbf{h})$ & 644.4 & 9.2 \\
\hline Frequency $(\mathbf{H z})$ & 9.2 & \\
\hline
\end{tabular}




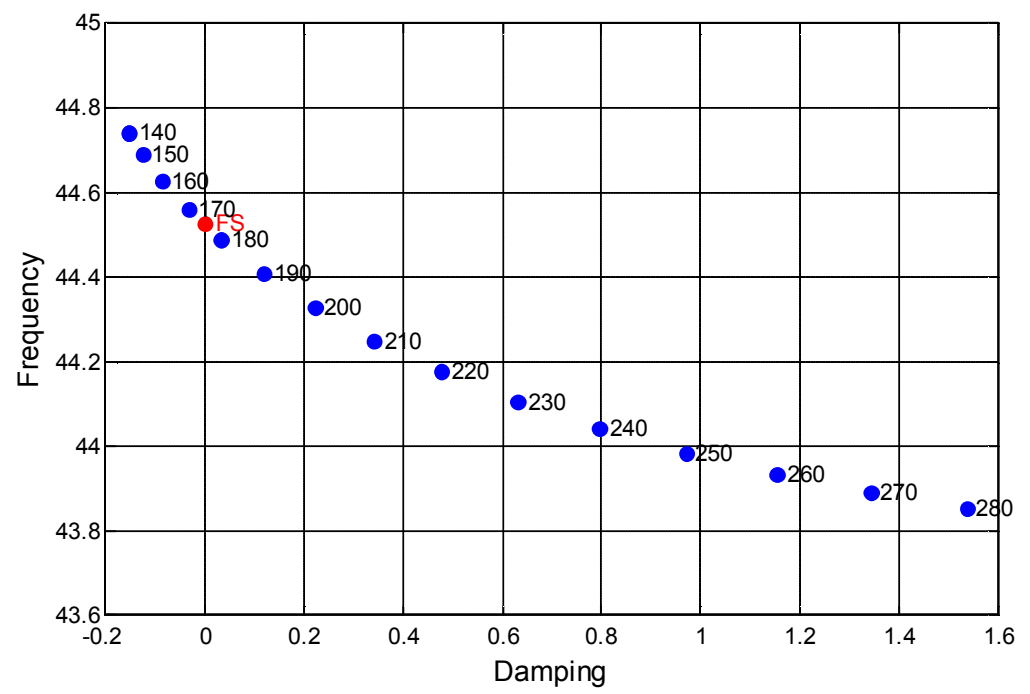

Fig 5.7 Flutter analysis by the EB beam structural model and the FS dynamic inflow unsteady aerodynamics model for the main wing 


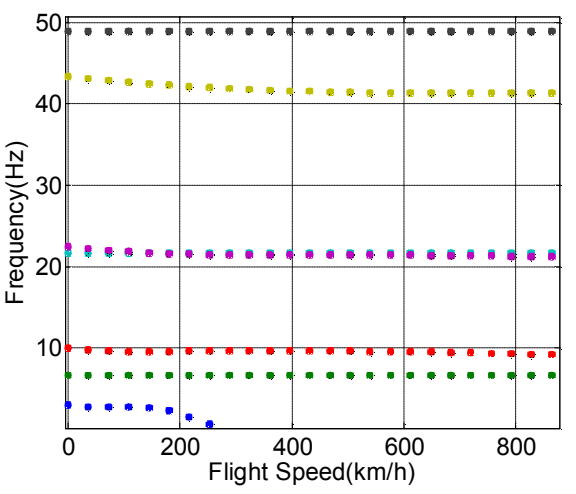

(a) Frequency plot by the EB beam structural model for the main wing

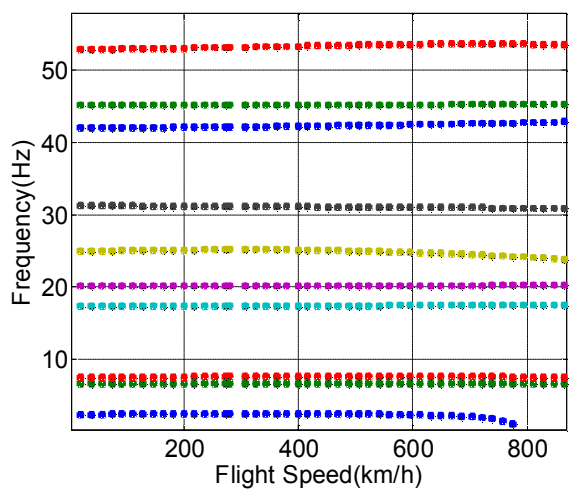

(c) Frequency plot by the 3-D FEM model for the main wing

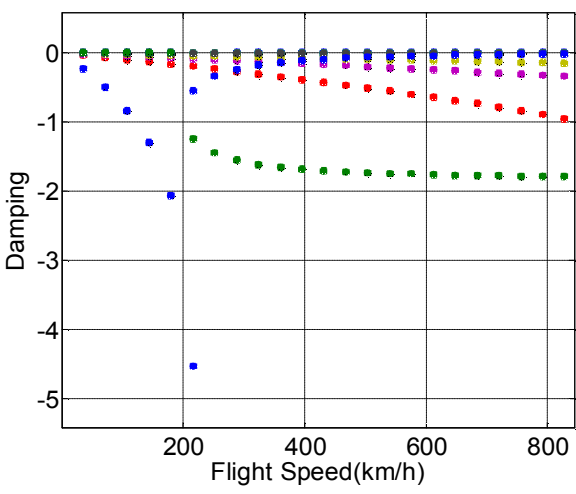

(b) Damping plot by the EB beam structural model for the main wing

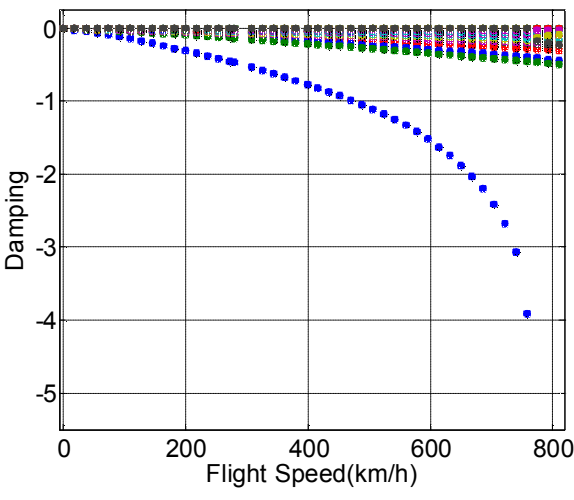

(d) Damping plot by the 3-D FEM model for the main wing

Fig 5.8 Damping and Frequency plots by the 3-D FEM and the EB beam structural model for the main wing at $\mathrm{M} 0.5$, non-matched flutter solution 


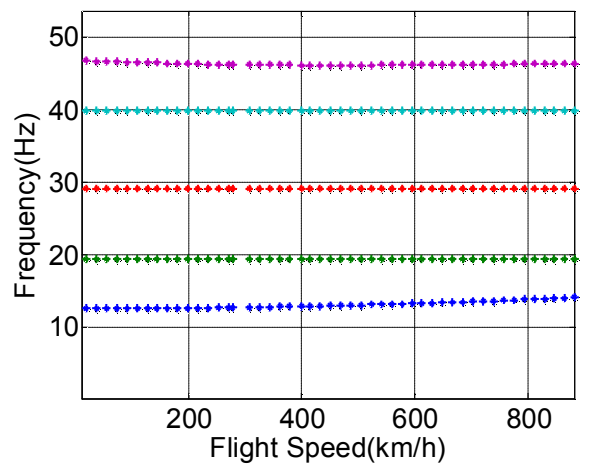

(a) Frequency plot by the 3-D FEM model for the tail wing

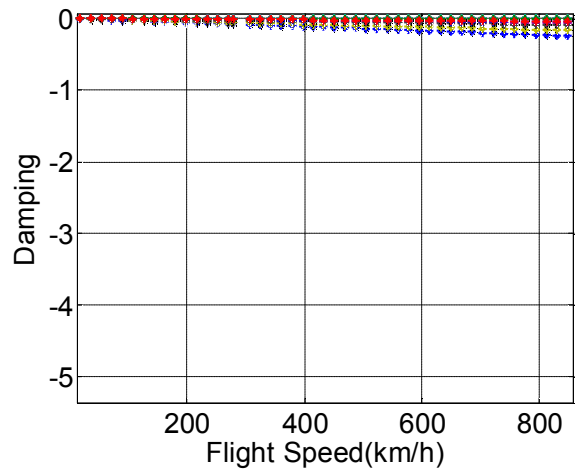

(b) Damping plot by the 3-D FEM model for the tail wing

Fig 5.9 Damping and Frequency plots by the 3-D FEM model for the tail wing at $M 0.5$, non-matched flutter solution 


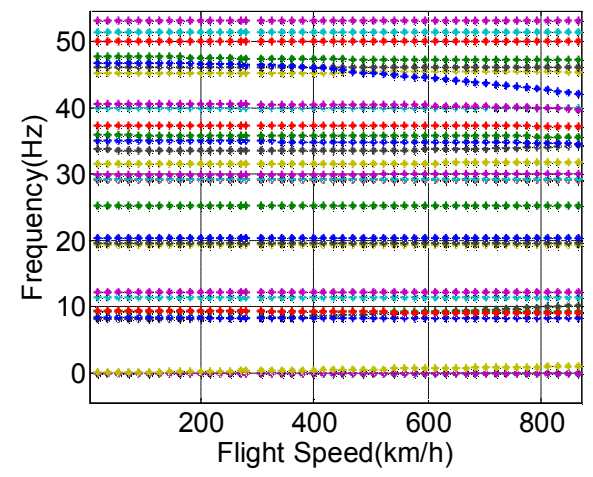

(a) Frequency plot by the 3-D FEM model for the complete aircraft

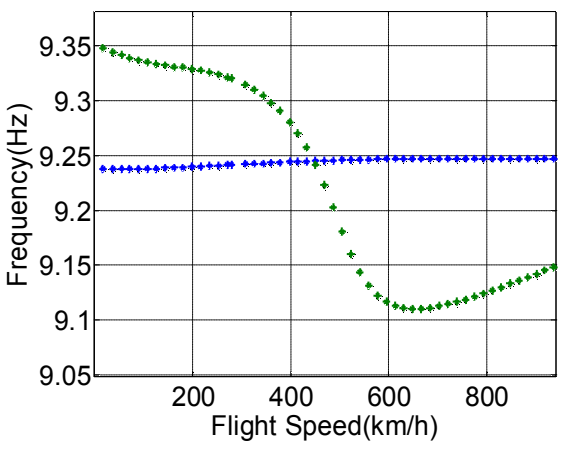

(c) Auxiliary Frequency plot the 3-D FEM model for the complete aircraft

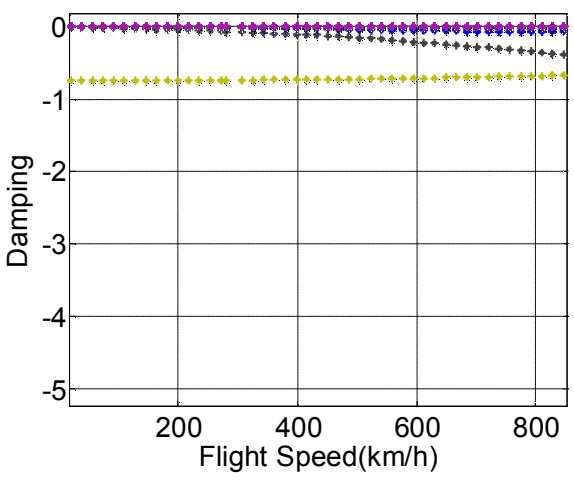

(b) Damping plot by the 3-D FEM model for the complete aircraft

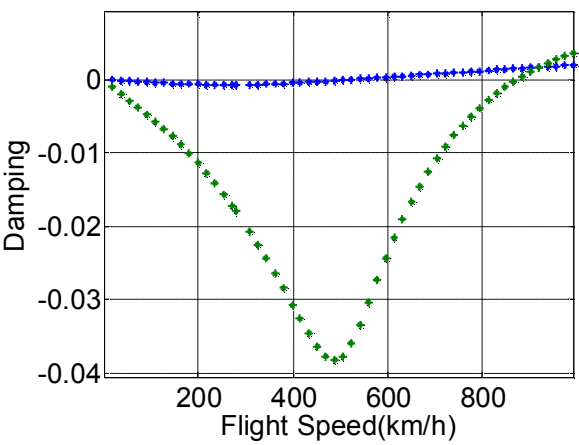

(d) Auxiliary Damping plot the 3-D FEM model for the complete aircraft

Fig 5.10 Damping and Frequency plots by the 3-D FEM for the complete aircraft at $M 0.5$, non-matched flutter solution 


\section{Chapter 6. Conclusion and Future Works}

\subsection{Summary}

In this research, an aeroelastic analysis of an aircraft with high aspectratio wings, developed for medium altitude and long endurance capabilities, was performed. In order to accurately and thoroughly analyze this aircraft and its inherent qualities, various structural and aerodynamic models were developed, implemented, and analyzed. To begin this analysis, a cross-sectional analysis was conducted by using VABS. This was complimented by the high aspect-ratio wing model, which was modeled by an Euler-Bernoulli beam structural model, a geometrically non-linear model, as well as a three-dimensional finite element model. Additionally, a structural analysis of the main wing and tail wing was performed. Through this analysis, the static deflection was extracted, and the natural frequencies and mode shapes were validated and compared with the threedimensional finite element model. The results obtained through this comparison demonstrated a strong and accurate correlation between the different models, in terms of both the natural frequency and mode shapes. These figures correlated with striking accuracy; for example, the natural frequencies of the Euler-Bernoulli beam structural model showed a 
maximum difference of $10 \%$ from those obtained through the threedimensional finite element model.

In addition to this, flutter results of the main wing were obtained through combining the Euler-Bernoulli beam structural model and two different aerodynamic models; the DLM model in ZAERO as well as the finite state dynamic inflow unsteady aerodynamics model in Matlab. The flutter result for the main wing was validated with three-dimensional finite element-based aeroelastic analysis. Hence, the results that were obtained throughout the analysis of the Euler-Bernoulli beam structural model were close to those predicted by the three-dimensional finite element model. For the main wing, there was only a difference of $7.94 \%$ in terms of the flutter speed, and only $2 \%$ disparity was observed in the case of the tail wing. What's more, further developments within the geometrically non-linear beam model were suggested to give a more accurate, succinct, and thorough representation of and comparison between the different models. The aforementioned model, would account for several important factors which include the trim effect and especially an ability to overcome any possible limitations that are the result of the use of the linearity.

\subsection{Future Works}

The geometrically non-linear beam model that has been used along this 
work is currently undergoing further developments. It is aimed to adapt the used formulation, which was originally suited for rotary wings aeroelasticity, to MALE aircraft's wing flutter analysis. After evaluating and properly implementing this structural model, the relevant finite state dynamic inflow unsteady aerodynamics are being adapted to allow for a combination of both which would provide another aeroelastic analysis tool for analysis of a high aspect-ratio wing. Although the effects of the nonlinearities is likely to appear in the case of a very stiff structure, chiefly because of the high value of the aspect-ratio of the wing that is the center of this research, analyzing the flutter based on that approach remains challenging and complex. Developing and validating the present geometrically non-linear structural model would allow for a simpler and more complete investigation of the non-linear aeroelastic phenomena that are related to it. All in all, the reduction of the problem's complexity and computation times without any loss of exactness will be kept as the main guideline towards the completion of the aforementioned step.

Another chief goal of this research remains to provide an analysis of the interaction between full six rigid-body degrees of freedom and aeroelastic modes. This very issue is believed to be a core component in understanding and enhancing the safety of unmanned aircraft. Several notable incidents in these types of aircraft, such as the HELIOS aircraft crash, only serve to illustrate the gravity and urgency of this issue [43]. 
Consequently, an important feature and future objective of the current thesis will be to perform the analysis and simulation of the flight dynamic response considering six rigid-body degrees of freedom. By adopting that approach, the flutter analysis would be more accurate and allow for other important factors such as the trim impact to be accounted for. Over the last decade, few researches tried to develop the following analysis despite its increased accuracy and accountability. Without a doubt, the analysis of the response of MALE aircraft, including those that account for the six degrees of freedom, will only help to provide a clearer and more accurate study of MALE aircraft as a whole. 


\section{References}

[1] Civil Aviation Authority, United Kingdom. "Unmanned Aerial Vehicle Operations in UK Airspace - Guidance.” CAP 722. 12 November 2004.

[2] Civil Aviation Safety Authority, Australia. "Unmanned Aircraft and Rocket Operations." CASR Part 101. January 2003.

[3] Friedmann, P., "Renaissance of Aeroelasticity and Its Future," Journal of Aircraft, Vol. 36, No. 1, pp. 105 - 121, Jan. - Feb., 1999

[4] Livne, E., "Future of Airplane Aeroelasticity," Journal of Aircraft, Vol. 40, No. 6, pp. 1066 - 1092, Nov. - Dec., 2003.

[5] Van Schoor M., Zerweckh S., and Von Flotow A., “Aeroelastic Stability and Control of a Highly Flexible Aircraft", AIAA-89-1187-CP, Proceedings of 30th AIAA / ASME / ASCE / AHS / ASC Structures, Structural Dynamics and Materials Conference, Mobile, AL, Apr. 3 - 5, 1989.

[6] Patil, M., Hodges, D., and Cesnik, C., "Nonlinear Aeroelasticity and Flight Dynamics of High-Altitude Long-Endurance Aircraft,” Journal of Aircraft, Vol. 38, No. 1, pp. 88 - 94, Jan 2001.

[7] Patil, M., Hodges, D., and Cesnik, C., "Nonlinear Aeroelastic Analysis of Complete Aircraft in Subsonic Flow" Journal of Aircraft, Vol. 37, No. 5, pp. $753-760$, Sep. - Oct., 2000. 
[8] Hodges D., and Dowell E., "Nonlinear Equations of Motion for Elastic Bending and Torsion of Twisted Non-uniform Blades," NASA TN D $7818,1974$.

[9] Chang, C., Hodges, D., and Patil, M., "Flight Dynamics of Highly Flexible Aircraft”, Journal of Aircraft, Vol. 45, No. 2, pp. 538 - 545, Mar. Apr., 2008.

[10] Bisplinghoff, R. L., Ashley, H., and Halfman, R. L. "Aeroelasticity." Addison-Wesley, Cambridge, 1995

[11] Fung, Y. C., "An Introduction to the Theory of Aeroelasticity." Dover, NewYork, 1969

[12] Woolston, D. S., Runyan, H. L., and Andrews, R. E., "An Investigation of Certain Types of Structural Nonlinearities on Wing and Control Surface Flutter," Journal of Aeronautical Sciences, Vol. 24, pp. 57 63,1957

[13] Shen, S. F., "An Approximate Analysis of Nonlinear Flutter Problems." Journal of Aeronautical Sciences, Vol. 26, pp. 25-32, 1959

[14] Kryloff, N., and Bogoliuboff, N., "Introduction to Nonlinear Mechanics.", Translation by Solomon Lifschitz, Princeton University Press, 1974.

[15] Hodges, D. H., and Dowell, E. H., "Nonlinear Equations of Motion for the Elastic Bending and Torsion of Twisted Nonuniform Rotor Blades." NASA TN D-7818, 1974. 
[16] Lee, B. H. K., and Leblanc, P., "Flutter Analysis of a TwoDimensional Airfoil with Cubic Nonlinear Restoring Force." National Research Council of Canada, NAE-AN-36, NRC No. 25438, 1986

[16] Lee, B. H. K., and Leblanc, P., "Flutter Analysis of a TwoDimensional Airfoil Containing Structural Nonlinearities." National Research Council of Canada, LR-618, NRC No. 27833, 1987

[17] Price, S. J., Alighanbari, H., and Lee, B. H. K., "The Aeroelastic Response of a Two-Dimensional Airfoil with Bilinear and Cubic Structural Nonlinearities." Journal of Fluids and Structures, Vol.9, pp. 75-193, 1995

[18] Lee, B. H. K., Gong, L., and Wong, Y. S., "Analysis and Computation of Nonlinear Dynamic Response of a Two-Degree-of-Freedom System and its Application in Aeroelasticity." Journal of Fluids and Structures, Vol.11, pp.225-246, 1997

[19] Strganac, T. W., and Mook, D. T., "A Numerical Model of Unsteady Subsonic Aeroelastic Behavior." AIAA Journal, Vol. 28, No. 5, pp. $903-$ 909, 1990.

[20] Hall, B. D., Preidikman, S., Mook, D. T., and Nayfeh, A. H., "Novel Strategy for Suppressing the Flutter Oscillations of Aircraft Wings." AIAA Journal, Vol. 39, No. 10, pp. 1843-1850, 2001

[21] Preidikman, S., and Mook, D. T., "Time-Domain Simulations of Linear and Nonlinear Aeroelastic Behavior." Journal of Vibration and Control, Vol. 6, No. 8, pp. 1135-1175, 2000 
[22] Patil, M. J., Hodges, D. H., and Cesnik, C. E. S., "Limit-Cycle

Oscillations in High Aspect ratio Wings." Journal of Fluids and Structures, Vol. 15, pp. 107-132, 2001

[23] Tang, D., and Dowell, E. H., "Experimental and Theoretical Study on Aeroelastic Response of High-Aspect-Ratio Wings." AIAA Journal, Vol. 39, No. 8, pp. 1430-1441, 2001

[24] Tang, D., and Dowell, E. H., "Experimental and Theoretical Study of Gust Response for High-Aspect-Ratio Wings." AIAA Journal, Vol. 40, No. 3, pp. 419-429, 2002

[25] Tang, D., and Dowell, E. H., "Effects of Geometric Structural Nonlinearity on Flutter and Limit Cycle Oscillations of High-Aspect-Ratio Wings." Journal of Fluids and Structures, Vol. 19, pp. 291-306, 2004.

[26] Patil, M. J., and Hodges, D.H., "On the importance of aerodynamic and structural geometrical nonlinearities in aeroelastic behavior of highaspect-ratio wings." Journal of Fluids and Structures, Vol. 19, pp. 905-915, 2004.

[27] Palacios, R., and Cesnik, C., "Static Nonlinear Aeroelasticity of Flexible Slender Wings in Compressible Flow", AIAA-2005-1945, 46th AIAA / ASME /ASCE / AHS /ASC Structures, Structural Dynamics, and Materials Conference, Austin, TX, Apr. 18 - 21, 2005.

[28] Peters D., and Cao W., "Finite State Induced Flow Models Part I: Two-Dimensional Thin Airfoil”, Journal of Aircraft, Vol. 32, No. 2, pp. 313 
- 322, Mar. - Apr., 1995.

[29] Cesnik, C., and Brown, E., "Modeling of High Aspect Ratio Active Flexible Wings for Roll Control”, 43rd AIAA / ASME / ASCE / AHS / ASC Structures, Structural Dynamics, and Materials Conference, Denver, CO, Apr. $22-25,2002$.

[30] Su, W., and Cesnik, C., "Dynamic Response of Highly Flexible Flying

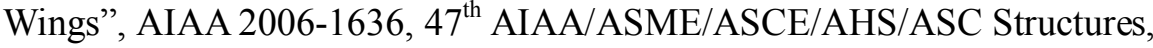
Structural Dynamics, and Materials Conference, Rhode Island, May. 1-4, 2006.

[31] Shearer, C., and Cesnik, C., "Nonlinear Flight Dynamics of Very Flexible Aircraft," Journal of Aircraft, Vol. 44, No. 5, pp. 1528 - 1545, Sep. - Oct., 2007

[32] Su, W., "Coupled Nonlinear Aeroelasticity and Flight Dynamics of Fully Flexible Aircraft," Ph.D Dissertation, University of Michigan, Ann Arbor, MI, 2008.

[33] Cesnik, C. E. S. and Hodges, D. H., "VABS: A New Concept for Composite Rotor Blade Cross-Sectional Modeling," Journal of the American Helicopter Society, Vol. 42, No. 1, pp. 27-38, 1997.

[34] Fung, Y.C, “An Introduction to the Theory of Aeroelasticity”. Dover Publications, Inc., New York, 1993.

[35] Rodden,W. P., "The Development of the Doublet-Lattice Method," Proceedings of the CEAS/International Forum on Aeroelasticity and 
Structural Dynamics, Vol. 2, pp. 1-7, June 1997

[36] Albano, E., and Rodden,W. P., “A Doublet-Lattice Method for Calculating the Lift Distributions on Oscillating Surfaces in Subsonic Flows,” AIAA Journal, Vol. 7, No. 2, pp. 279-285, 1969

[37] Giesing, J. P., Kalman, T. P., and Rodden, W. P., "Subsonic Unsteady Aerodynamics for General Configurations, Part I—Direct Application of the Non-Planar Doublet-Lattice Method," Air Force Flight Dynamics Lab., Air Force Systems Command, Rept. AFFDL-TR-71-5, Pt. 1, WrightPatterson AFB, OH, April 1972.

[38] Theodorsen, T., "General Theory of Aerodynamic Instability and the Mechanism of Flutter," NACA TR No. 496, May 1934.

[39] Peters, D. A. and Johnson, M. J., "Finite-State Airloads for Deformable Airfoils on Fixed and Rotating Wings," Symposium on Aeroelasticity and Fluid/Structure Interaction, Proceedings of the Winter Annual Meeting. ASME, November 6-11, 1994.

[40] Peters, D. A., “Two-dimensional incompressible unsteady airfoil theory-An overview," Journal of Fluids and Structures, Vol. 24, No. 3, pp. $295-312,2008$.

[41] Isaacs, R., "Airfoil Theory for Flows of Variable Velocity," Journal of the Aeronautical Sciences, Vol. 12, No. 1, pp. 113-117, 1945.

[42] Chung, C. H., Shin, S. J., and Kim, T., "Development and Verification of an Aircraft Flutter Analysis with Uncertainty," AIAA-2007-2369, 48th 
AIAA/ASME/ASCE/AHS/ASC Structures，Structural Dynamics, and Materials Conference, Honolulu, Hawaii, Apr. 23-26, 2007.

[43] Noll, T. E., Brown, J. M., Perez-Davis, M. E., Ishmael, S.D., Tiffany G.C., and Gaier, M., "Investigation of the Helios Prototype Aircraft Mishap," Tech. rep., NASA, January 2004. 


\section{요약 (국문초록)}

본 논문에서는 고세장비 항공기 날개의 공탄성 해석을 시도하였다. 이 를 위하여 다양한 구조 모델이 시도되고 수행되었다. 전통적인 접근법 은 Euler Bernoulli 보 구조 모델을 기반으로 하고 있으며, 본 논문에서 는 추가로 기하학적 비선형 보 모델을 적용하였다. 소개된 보 모델의 구조 해석 결과는 3차원 NASTRTAN 유한요소 모델 해석결과와 비교하 였고 비교 결과는 두 모델의 고유진동수와 고유모드 상에서 유사한 결 과가 얻어짐을 보여주었다. 비정상 공력의 경우 유한 상태 동적 유입류 비정상 공력과, Doublet Lattice 기법에 기반을 두고있는 ZAERO 상용해 석 프로그램을 바탕으로 도출된 비정상 공력등의 두 가지의 방법이 사 용되었다. 또한 플러터 해석을 위하여 위의 두 공력 해석 결과를 비교 하였다. 플러터 해석을 위하여 Euler Bernoulli 보의 고유 모드 및 고유 진동수 해석 결과와 3차원 NASTRAN유한 요소 모델의 구조 해석 결과 를 ZAERO 상용프로그램과 결합을 수행하였다. 향후 연구에서는, 강체 비행 모드와 공력탄성학 모드를 함께 고려한 플러터 해석을 수행하는 것이 요구된다.

주요어: 고세장비 날개, Euler Bernoulli 보 모델, 3 차원 유한 요소 모 델,기하학적 비선형 보 모델, Doublet lattice method, 유한상 태 동적 유입류 비정상 공기력 모델, 플러터 해석

학 번: 2011-22890 